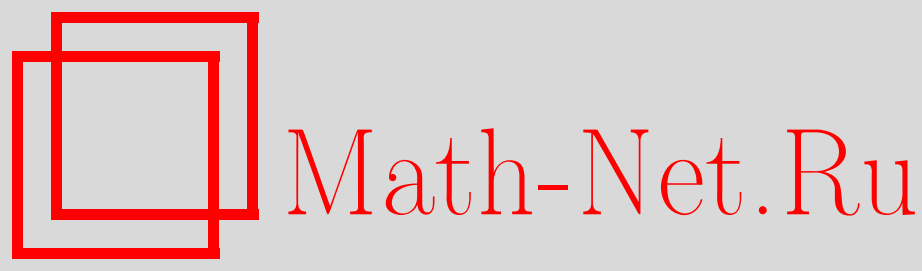

Х. Итояма, А. Д. Миронов, А. Ю. Морозов, Сшивка ветвей непертурбативного конформного блока на его дивизоре сингулярностей, ТМФ, 2015, том 184, номер 1, 3-40

DOI: https://doi.org/10.4213/tmf8856

Использование Общероссийского математического портала Math-Net.Ru подразумевает, что вы прочитали и согласны с пользовательским соглашением http://www . mathnet.ru/rus/agreement

Параметры загрузки:

IP : 3.82 .47 .9

26 апреля 2023 г., $16: 22: 27$

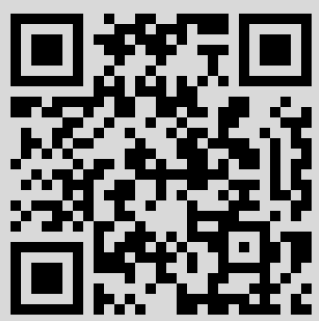




\title{
СШИВКА ВЕТВЕЙ НЕПЕРТУРБАТИВНОГО КОНФОРМНОГО БЛОКА НА ЕГО ДИВИЗОРЕ СИНГУЛЯРНОСТЕЙ
}

\begin{abstract}
Конформный блок является функцией многих переменных, которая обычно представляется в виде формального ряда с коэффициентами, являющимися некоторыми матричными элементами в киральной алгебре (т.е. в алгебре Вирасоро). Непертурбативный конформный блок есть многозначная функция, определенная на пространстве размерностей, со многими ветвями и, возможно, с дополнительными свободными параметрами, не видными на пертурбативном уровне. Обсуждаются дополнительные усложнения непертурбативного описания, возникающие по причине того, что все наилучшим образом изученные примеры лежат на локусе сингулярностей в пространстве модулей (на дивизоре коэффициентов или просто в нулях детерминанта Каца). Типичным примером является точка Ашкина-Теллера, где имеются по меньшей мере два непертурбативных выражения, заданных эллиптическим интегралом Доценко-Фатеева и знаменитой формулой Ал. Замолодчикова в терминах тета-констант. Эти выражения различны. Ситуация в некотором роде напоминает модель Изинга и другие точки минимальных моделей.
\end{abstract}

Ключевые слова: двумерные конформные теории, конформные блоки.

DOI: $10.4213 / \operatorname{tmf} 8856$

\section{1. ВВЕДЕНИЕ}

Конформные блоки являются центральным объектом в двумерных конформных теориях [1] - они представляют собой голоморфные составляющие корреляционных функций, так что последние разлагаются в билинейные комбинации конформных блоков с различными внутренними (промежуточными) размерностями. Другой

* Department of Mathematics and Physics, Osaka City University, Osaka, Japan.

E-mail: itoyama@sci.osaka-cu.ac.jp

${ }^{\dagger}$ Osaka City University Advanced Mathematical Institute (OCAMI), Osaka, Japan

${ }_{\ddagger}^{\ddagger}$ Физический институт им. П. Н. Лебедева РАН, Москва, Россия. E-mail: mironov@lpi.ru

${ }^{\S}$ Институт теоретической и экспериментальной физики, Москва, Россия.

E-mail: morozov@itep.ru

๑ Московский физико-технический институт, Долгопрудный, Московская обл., Россия 
ингредиент теории - структурные константы, определяющие коэффициенты этих разложений (для многих целей удобно не включать их в нормировку конформных блоков, которая выбирается тогда некоторым иным образом, более удобным с точки зрения комплексного анализа). Корреляционные функции можно разложить несколькими разными способами, и соответствующие конформные блоки связаны линейными преобразованиями, которые называются модулярными. При помощи формализма свободных фермионов [2] определенные производящие функции интерпретируются как $\tau$-функции обычных интегрируемых систем и иерархий типа КП/Тоды, а обобщение этого формализма на модели ВЗНВ [3], [4] должно обеспечить их описание как неабелевых $\tau$-функций из работ [5].

Много лет назад была дана интерпретация конформных блоков как состояний в гильбертовом пространстве трехмерной теории Черна-Саймонса [6], и таким образом был предоставлен важный ингредиент современных квантово-полевых подходов [7]-[11] к теории узлов [12]. Позже соотношения Алдая-Гайотто-Тачикавы (АГТ) [13]-[19] позволили дать еще одну интерпретацию конформных блоков и их прямых $q$-деформаций [20]-[22] как сумм по инстантонам в соответственно четырехи пятимерных теориях Янга-Миллса с расширенной суперсимметрией [23]-[25]. Соответствие АГТ оказалось полезным в обоих направлениях: скажем, при использовании сумм по инстантонам для анализа минимальных моделей [15], [26], [27] и для использования решения Ал. Замолочикова [28] при анализе инстантонных вкладов в теории Зайберга-Виттена в конформной точке [29], [30]. Все эти применения к квантовой теории поля в разных размерностях объясняют центральную роль конформных блоков в теоретической физике и необходимость их тщательного изучения. Оно было достаточно далеко продвинуто, но, к сожалению, не закончено Ал. Замолодчиковым [28], [31], [32].

В настоящей работе мы концентрируемся на, возможно, простейшем нетривиальном из всех конформных блоков: четырехточечном сферическом блоке, который обычно определяют формальным рядом по двойному отношению $x=\frac{\left(x_{2}-x_{1}\right)\left(x_{3}-x_{4}\right)}{\left(x_{3}-x_{1}\right)\left(x_{2}-x_{4}\right)}$ четырех точек на римановой сфере:

$$
B(x)=\sum_{k=0}^{\infty} B_{k} x^{k} .
$$

Коэффициенты $B_{k}$ зависят от четырех внешних размерностей $\Delta_{1}, \ldots, \Delta_{4}$, от одной внутренней размерности $\Delta$ и от центрального заряда $c$ :

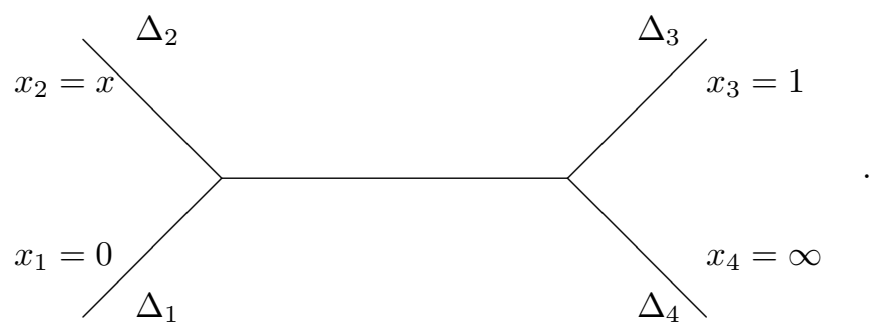

Ряд (1.1) не всюду сходится, таким образом, возникает вопрос, какова функция $\mathcal{B}(x)$, для которой $B(x)$ является разложением в формальный ряд в окрестно- 
сти точки $x=0$. Мы называем такую функцию непертурбативным конформным блоком и далее приводим вкратце ее уже известные свойства.

Имеется вера в то, что как функция от $x$ четырехточечный сферический конформный блок является аналитической функцией, ветвящейся в точности в трех точках $0,1, \infty$ и не имеющей существенных особенностей. Более того, в рациональных конформных теориях ветвления имеют конечный порядок, таким образом, данные конформные блоки в действительности являются функциями Белого [33], которые появляются при рассмотрении “детских рисунков" Гротендика и равносторонних триангуляций. Разветвленные накрытия пространства $\mathbb{C P}^{1}$, описывающиеся функциями Белого, суть арифметические кривые, и их описание в терминах конформных моделей и рациональных размерностей представляет собой новую интересную проблему, связанную с описанием универсального пространства модулей [34], [35] и современной теорией чисел Гурвица [36] и $\tau$-функций Гурвица [37], [38].

Возвращаясь к непертурбативной функции $\mathcal{B}(x)$, заметим, что сходимость разложения (1.1) по степеням $x$ неравномерна по другим параметрам $\left(\Delta_{i}, \Delta, c\right)$, что делает сумму ряда как функцию всех этих переменных весьма изысканной. Функция $\mathcal{B}(x)$, конечно, нетривиальна: она имеет много ветвей и изменяется при модулярном преобразовании. Более того, это изменение может быть представлено интегральным преобразованием по внутренней размерности $\Delta$ :

$$
\begin{aligned}
\mathcal{B}_{\Delta}(1-x) & =\sum_{\Delta^{\prime}} \mathcal{M}_{\Delta}^{\Delta^{\prime}} \mathcal{B}_{\Delta^{\prime}}(x), \\
\mathcal{B}_{\Delta}\left(\frac{x}{x-1}\right) & =\sum_{\Delta^{\prime}} \mathcal{N}_{\Delta}^{\Delta^{\prime}} \mathcal{B}_{\Delta^{\prime}}(x) .
\end{aligned}
$$

Непертурбативные модулярные ядра $\mathcal{M}_{\Delta}^{\Delta^{\prime}}$ и $\mathcal{N}_{\Delta}^{\Delta^{\prime}}$ изучены значительно лучше, чем функция $\mathcal{B}_{\Delta}(x)$. Сам факт, что существует модулярное ядро, не зависящее от $x$, совершенно нетривиален. Это случается, только если промежуточная (внутренняя) размерность $\Delta$ выбирается в качестве параметра интегрального преобразования (ничего подобного не происходит, если мы пытаемся использовать, скажем, одну из размерностей $\Delta_{i}$ или заряд $c$ ), и отражает свойство ассоциативности операторного разложения (OP) в конформной теории поля (на него также ссылаются как на дуальность) или коумножения в алгебре Вирасоро, причем модулярное ядро является аналогом коэффициентов Рака (или $6 j$-символов) в теории конечномерных алгебр Ли.

В контексте квантовой теории поля и, в частности, в конформной теории обычно рассматривают заданный набор полей, т. е. фиксируют набор внешних размерностей $\Delta_{i}$, в то время как $\Delta$ остается произвольной: обычным в этом контексте является изучение зависимости от $\Delta$, и не столь принято обращать равное внимание на зависимости от $\Delta_{i}$ или $c$, т.е. на точку в "пространстве теорий". Тем не менее в рамках современных струнных подходов более чем естественно прямо обсуждать функцию $\mathcal{B}(\mu \mid x)$ на полном пространстве $\mathcal{M}$ шести комплексных параметров $\mu=\left\{\Delta_{i}, \Delta, c\right\}$, без выделения одномерного "физического среза" $\mathcal{P} \subset \mathcal{M}$, где $\Delta_{i}, c=$ const. На самом деле конформный блок должен аналитически продолжаться не только по $x$, но также по всем этим шести дополнительным параметрам $\mu$. В частности, имеется уверенность в том, что модулярное ядро, которое живет на физическом срезе и потому уже привлекло определенное внимание, обладает непертурбативными поправками как функция от переменных $\Delta$ и $\Delta^{\prime}$ [39]. 
Таким образом, наша цель в настоящей работе - привлечь внимание к непертурбативному конформному блоку $\mathcal{B}(\mu \mid x)$ не только на физическом срезе $\mathcal{P} \subset \mathcal{M}$, но везде, чтобы идентифицировать природу и сущность этого важного класса специальных функций. Оказывается, что даже на первом шаге в этом направлении встречаются интересные детали, и это должно стимулировать дополнительное внимание к проблемам такого типа.

Имеются специальные случаи внешних размерностей и центрального заряда, когда, как кажется, многое известно о $\mathcal{B}(x)$, и естественно начать наше рассмотрение с этого места.

А. Для вырожденных модулей Верма существуют условия нуль-векторов, которые подразумевают определенные дифференциальные уравнения для конформного блока как функции от $x$ [1]. Порядок уравнения определяется уровнем нуль-вектора, и во всех этих случаях он, во-первых, конечен и, во-вторых, больше единицы. Второе свойство означает, что у конформного блока $\mathcal{B}(x)$ имеются различные ветви, и он в действительности живет на нетривиальной римановой поверхности в $x$-пространстве. Первое свойство означает, что имеется только конечномерное семейство решений, в то время как можно думать, что только один из шести параметров $\mu$ (а именно конкретная внешняя размерность, скажем, $\Delta_{2}$ ) фиксирован условием нуль-вектора, и функция оставшихся пяти параметров должна быть решением.

В. Конформный блок имеет представление Доценко-Фатеева в терминах многократных (обобщенных гипергеометрических) интегралов Сельберга, аналитически продолженных по числам $N_{1}, N_{2}$ интегрирований [17]. Однако при фиксированных $N_{1}$ и $N_{2}$ интегралы Сельберга представляют собой обычные интегралы и, таким образом, могут исследоваться, как непертурбативные объекты, обычными методами комплексного анализа. Это очевидная возможность, но она аккуратно не изучалась, поскольку такой “интегральный срез" $\mathcal{I}$, определенный условием $N_{1}, N_{2}=$ const, в некотором смысле трансверсален физическому срезу $\mathcal{P}$, а на конформные блоки в трансверсальных направлениях никогда внимательно не смотрели.

C. Наконец, имеется знаменитая дуальность Замолодчикова [40], применимая к очень специальной модели (Ашкина-Теллера) с $c=1$ и $\Delta_{i}=1 / 16$. Суть в том, что операторы такой размерности создают сингулярности типа корня для свободных фермионов (коих два в теории с $c=1$ ), и, таким образом, коррелятор полей $V_{1 / 16}$ в некоторых точках может идентифицироваться со статистической суммой свободных фермионов (модель Изинга) на разветвленном накрытии первоначального пространства-времени. В специальном случае четырехточечного коррелятора мы получаем тор с зависящим от $x$ модулярным параметром $\tau$ и, в результате, фантастическую формулу для конформного блока на замолодчиковском срезе $\mathcal{Z} \subset \mathcal{M}$ c $\Delta_{i}=1 / 16, c=1$ :

$$
\mathcal{B}_{\Delta}(x)=\operatorname{Tr}_{\text {free fermions }} e^{i \pi \tau L_{0}}=\frac{q^{\Delta}}{\theta_{00}(q)},
$$

где

$$
q=e^{i \pi \tau}, \quad x=\frac{\theta_{10}^{4}(q)}{\theta_{00}^{4}(q)}, \quad \theta_{00}(q)=\sum_{n=-\infty}^{\infty} q^{n^{2}}=\prod_{m=1}^{\infty}\left(1-q^{2 m}\right)\left(1+q^{2 m-1}\right)^{2} .
$$

Это опять хорошо определенная непертурбативная формула, столь известная, поскольку она записывается на физическом срезе. 
В настоящей работе мы обсуждаем приведенные выше примеры и взаимоотношения между ними. Наше основное наблюдение состоит в том, что как минимальные модели (типичным является пример, где все внешние состояния являются нуль-векторами), так и модель Замолодчикова при выделенном значении $\Delta=1 / 4$ лежат, как оказывается, на локусе сингулярностей $\mathcal{L} \subset \mathcal{M}$, где коэффициенты конформных блоков являются отношениями двух обращающихся в ноль функций и, таким образом, они неоднозначно определены. Это тривиальное, но ранее недооцененное явление, поскольку его нельзя заметить на физическом срезе: $\mathcal{L}$ и $\mathcal{P}$ трансверсальны, когда пересекаются. Весьма интересно понять, что случается в этих пересечениях. Более того, вероятно, будущие исследования этих сингулярностей помогут понять непертурбативную зависимость конформных блоков от $\mu$, которая важна для того, чтобы пролить свет на несколько мистические непертурбативные выражения для модулярных ядер (постулированные отождествлением [41], [40] матриц Рака алгебры Вирасоро с матрицами Рака специальных представлений группы $S L(2))$.

Чтобы понять это явление, нужно изучить конформный блок как формальный ряд по $x$ в окрестности сингулярностей. Сингулярности находятся в нулях Каца, т. е. всегда, когда удовлетворяются специальные соотношения между внутренней размерностью $\Delta$ и центральным зарядом $c$. Более конкретно, если взять конформные размерности в параметризации Доценко-Фатеева,

$$
\Delta=\alpha\left(\alpha-b+\frac{1}{b}\right), \quad c=1-6\left(b-\frac{1}{b}\right)^{2}
$$

то нули Каца у коэффициентов $B_{k}$ находятся в точках

$$
\left.\alpha=\frac{1}{2}\left(\frac{n-1}{b}-(m-1) b\right)\right),
$$

где $m$ и $n$ - любые целые ненулевые числа такие, что $m n \leqslant k$. Для того чтобы ответ был несингулярным, нужно потребовать, чтобы конформный блок имел добавочные нули в этих сингулярностях. Так как конформный блок является функцией от $x$, нужно это потребовать для каждого сингулярного $B_{k}$. В действительности, как мы увидим, нули Каца имеют вложенную структуру: как только $B_{k}$ сингулярен при некотором $k$, также сингулярны все $B_{l} \mathrm{c} l>k$. Достаточно удивительно, что, как только налагается условие обращения в ноль числителя коэффициента $B_{k}$ в нуле Каца, во всех $B_{l}$ с $l>k$ числители также становятся равными нулю! Более того, несмотря на то что ответ зависит от направления подхода к сингулярностям, он параметризуется всего одной произвольной постоянной.

Однако в рациональных теориях возникает новое явление: из-за рационального значения центрального заряда случайным образом появляются полюсы (нули Каца) более высокого порядка. Снова когда такой, допустим, двойной полюс возникает при некотором $x$, он также представлен во всех $B_{l}$ c $l>k$ до тех пор, пока он не станет полюсом третьего порядка и т.д. Однако это, вообще говоря, больше не имеет место для соответствующих нулей в числителе, которые необходимы, чтобы скомпенсировать эти полюсы: в числителях имеются обычно простые нули даже в рациональных конформных теориях, т.е. в этих случаях мы имеем настоящую сингулярность. Это означает, что в согласованной теории соответствующие структурные константы должны обращаться в ноль, что делает структуру рациональной конформной теории в окрестности сингулярности весьма изысканной. 
Замечательным исключением являются минимальные модели. В случае минимальных моделей ответ не зависит от направления подхода к сингулярности! Это выделяет такие модели и делает их однозначно определенными.

В настоящей работе мы иллюстрируем описанную картину конкретными примерами модели Ашкина-Теллера и минимальных моделей. Заметим, что модель Ашкина-Теллера является единственным примером, к настоящему моменту известным в явном виде, когда ответ неоднозначен вблизи сингулярности; другие известные примеры - минимальные модели, когда эта неоднозначность отсутствует.

\section{2. РЯДЫ И "НЕПЕРТУРБАТИВНЫЕ ЭФФЕКТЫ"}

Мы начинаем с общего обсуждения того, что такое непертурбативный конформный блок, т. е. того, что такое конформный блок вне его радиуса сходимости ${ }^{1)}$. Архетипический пример асимптотического пертурбативного ряда дается выражением

$$
F(x)=\sum_{n=0}^{\infty} n ! x^{n}
$$

Факториальный рост здесь обычно связан с тем фактом, что возмущение представлено иррелевантными операторами, и непертурбативный ответ должен иметь отличающуюся асимптотику при больших значениях полей.

Наиболее очевидный способ анализировать ряд (2.1) - интерпретировать факториалы как гамма-функцию и использовать ее интегральное представление, чтобы определить непертурбативную функцию

$$
\mathcal{F}(x)=\sum_{n=0}^{\infty} \Gamma(n+1) x^{n}=\int_{0}^{\infty}\left(\sum_{n=0}^{\infty}(z x)^{n}\right) e^{-z} d z=\int_{0}^{\infty} \frac{e^{-z} d z}{1-z x} .
$$

Этот ответ, однако, зависит от выбора контура, связывающего 0 и $\infty$, и неоднозначность - это просто вычет в сингулярности подынтегрального выражения: функция $\mathcal{F}(x)$ определена по модулю выражения

$$
\oint_{z=x^{-1}} \frac{e^{-z} d z}{1-z x} \sim \frac{e^{-1 / x}}{x}
$$

Альтернативный путь определить непертурбативную функцию $\mathcal{F}(x)$ - написать уравнение, которому удовлетворяет пертурбативный ряд. В случае (2.1) простейшим является уравнение первого порядка

$$
x \frac{d}{d x} x F(x)=\sum_{n=0}^{\infty}(n+1) ! x^{n+1}=F(x)-1 .
$$

\footnotetext{
1) Заметим здесь, что радиус сходимости конформного блока зависит от выбранных переменных. $\mathrm{K}$ примеру, по переменной $x$ этот радиус не превышает единицы, поскольку при $x=1$ имеется сингулярность. В то же время при использовании переменной $q=e^{\pi i \tau}$, связанной с $x$ формулой $x=\theta_{10}^{4} / \theta_{00}^{4}$, где

$$
\theta_{10}(q)=\theta_{10}(0 \mid \tau)=2 q^{1 / 4} \sum_{n=0} q^{n(n+1)}=2 q^{1 / 4} \prod_{m=1}^{\infty}\left(1-q^{2 m}\right)\left(1+q^{2 m}\right)^{2},
$$

ряды сходятся во всей области аналитичности функции $\mathcal{B}(x)$, как было показано Ал. Замолодчиковым [28].
} 
Решения этого уравнения зависят от свободной константы: мы можем добавить (с произвольным коэффициентом) решение однородного уравнения

$$
x^{2} \frac{d F_{0}}{d x}=(1-x) F_{0}(x) \Longrightarrow F_{0}(x)=\frac{e^{-1 / x}}{x},
$$

т. е. $\mathcal{F}(x)$ снова определена по модулю $x^{-1} e^{-1 / x}$. Небольшое отличие состоит в том, что коэффициент в первом подходе - произвольное целое число, в то время как во втором, т. е. когда непертурбативная функция определена как $D$-модуль, как решение некоторого линейного уравнения, коэффициент, естественно, может быть произвольным комплексным числом.

Конечно, в обоих случаях мы рассматриваем минимальное определение: непертурбативная функция может быть поднята на большее пространство модулей, к примеру, путем рассмотрения уравнений старшего порядка, у которых имеется больше решений. Тем не менее обычно не существует естественного пути избавиться от добавочных параметров; или, когда это удается сделать, должна быть некоторая дополнительная причина для такого ограничения. Таким образом, мораль на данной стадии такова: непертурбативная функция обычно зависит от дополнительных (скрытых) параметров.

Подчеркнем, что этот факт неявно присутствует в любом непертурбативном рассмотрении. Достаточно напомнить знаменитый пример инстантонов в теориях Янга-Миллса: они не только входят с типичным непертурбативным весом $e^{-1 / g^{2}}$, но и вносят дополнительный параметр, тета-угол, не наблюдаемый на пертурбативном уровне. На самом деле ограничение на вещественные углы (т. е. на унимодулярные свободные коэффициенты перед инстантонным вкладом) в некотором роде аналогично ограничению на целочисленные коэффициенты в вышеприведенном примере, другими словами, связано скорее с конкретным методом аналитического продолжения, чем с существом проблемы. Что в этом примере важно - это то, что он подчеркивает физическую значимость дополнительных (пертурбативно скрытых) параметров: они влияют на непертурбативную перенормировку [42], важность которой в наши дни общепризнана, и которая широко исследована с помощью теории Зайберга-Виттена [43]-[45].

В применении к конформным блокам это должно бы подразумевать, что $\mathcal{B}(\mu \mid x)$ на самом деле есть $\mathcal{B}(\mu|x| C)$, т. е. непертурбативный конформный блок зависит от дополнительных параметров $C$, не наблюдаемых на пертурбативном уровне и не представленных в первоначальном разложении (1.1), которое получено из теории представлений алгебры Вирасоро.

Однако нужно ожидать, что конформная теория является по существу свободной теорией [46]-[48], [4], следовательно, в ней нет места факториальному росту разложений по константам связи иррелевантных операторов. Таким образом, непертурбативные скрытые параметры не появляются в данном контексте, и все неоднозначности имеют иную природу. Как мы увидим, это делает конформные блоки легче познаваемыми, нежели непертурбативные эффекты в более общих взаимодействующих моделях квантовой теории поля. Как уже упоминалось, имеется вера в то, что разложение (1.1) в ряд по $x$ имеет конечные радиусы сходимости, и в этом его отличие от (2.1). 
На самом деле даже ряд (2.1), как недавно было показано [38], может быть естественным образом ассоциирован с определенной $\tau$-функцией иерархий КП/Тоды (возникающей в упрощенной задаче перечисления функций Белого [49]). Поскольку $\tau$-функции удовлетворяют квадратичным уравнениям Хироты [50], [2], [5], они не сохраняются при линейных преобразованиях. Это может дать новый интересный инструмент для ограничения неоднозначностей (возможно, даже обеспечить выбор $\theta=0$ в инстантонном вычислении, что приведет, таким образом, к новому типу решения тета-проблемы).

\section{3. ПЕРТУРБАТИВНОЕ РАЗЛОЖЕНИЕ КОНФОРМНОГО БЛОКА}

Мы начинаем с напоминания основных фактов о пертурбативном конформном блоке (1.1).

\section{1. Определение конформного блока для четырех примарных полей.} Базовое определение четырехточечного конформного блока приходит из билинейного разложения корреляционной функции четырех примарных полей [1]:

$$
\begin{gathered}
\left\langle V_{\Delta_{1}, \bar{\Delta}_{1}}\left(x_{1}, \bar{x}_{1}\right) V_{\Delta_{2}, \bar{\Delta}_{2}}\left(x_{2}, \bar{x}_{2}\right) V_{\Delta_{3}, \bar{\Delta}_{3}}\left(x_{3}, \bar{x}_{3}\right) V_{\Delta_{4}, \bar{\Delta}_{4}}\left(x_{4}, \bar{x}_{4}\right)\right\rangle= \\
=\sum_{\Delta, \bar{\Delta}} C_{12}^{\Delta, \bar{\Delta}} C_{34}^{\Delta, \bar{\Delta}} G_{\Delta}\left(\Delta_{1}, \Delta_{2}, \Delta_{3}, \Delta_{4} ; c ; x_{1}, x_{2}, x_{3}, x_{4}\right) \times \\
\times \bar{G}_{\bar{\Delta}}\left(\bar{\Delta}_{1}, \bar{\Delta}_{2}, \bar{\Delta}_{3}, \bar{\Delta}_{4} ; c ; \bar{x}_{1}, \bar{x}_{2}, \bar{x}_{3}, \bar{x}_{4}\right),
\end{gathered}
$$

где $C_{12(34)}^{\Delta, \bar{\Delta}}$ - структурные константы теории, которые иногда включают в определение конформного блока. Однако по сути структурные константы являются самостоятельными объектами, они определяют конкретную конформную теорию, и отдельной задачей является перечисление всех допустимых структурных констант (они должны удовлетворять дополнительным сложным соотношениям типа соотношений дуальности).

В то же время конформный блок $G_{\Delta}\left(\Delta_{1}, \Delta_{2}, \Delta_{3}, \Delta_{4} ; c ; x_{1}, x_{2}, x_{3}, x_{4}\right)$ является универсальной функцией четырех точек $x_{i}$, которая зависит от шести параметров - пяти размерностей и центрального заряда; он кодирует только свойства алгебры Вирасоро. На самом деле это нетривиальная функция, зависящая только от двойного отношения $x=\frac{\left(x_{2}-x_{1}\right)\left(x_{3}-x_{4}\right)}{\left(x_{3}-x_{1}\right)\left(x_{2}-x_{4}\right)}$ :

$$
\begin{gathered}
G_{\Delta}\left(\Delta_{1}, \Delta_{2}, \Delta_{3}, \Delta_{4} ; c ; x_{1}, x_{2}, x_{3}, x_{4}\right)=\left(\prod_{i<j} x_{i j}^{\zeta_{i j}}\right) B_{\Delta}(x)= \\
=\left(\prod_{i<j} x_{i j}^{\zeta_{i j}}\right) \sum_{k} x^{k} B_{k}\left(\Delta_{1}, \ldots, \Delta_{4} \mid \Delta, c\right) .
\end{gathered}
$$

Здесь $x_{i j} \equiv x_{i}-x_{j}$ и

$$
\begin{aligned}
& \zeta_{12}=\zeta_{13}=0, \quad \zeta_{14}=-2 \Delta_{1}, \quad \zeta_{23}=\Delta_{4} \Delta_{1}-\Delta_{2}-\Delta_{3}, \\
& \zeta_{24}=\Delta_{1}+\Delta_{3}-\Delta_{2}-\Delta_{4}, \quad \zeta_{34}=\Delta_{1}+\Delta_{2}-\Delta_{3}-\Delta_{4} \text {. }
\end{aligned}
$$

Заметим, что перестановки точек приводят к модулярным преобразованиям (1.2). $\mathrm{K}$ примеру, при перестановке $x_{1}$ и $x_{3}$ мы получим

$$
B\left(\Delta_{3}, \Delta_{2}, \Delta_{1}, \Delta_{4} \mid \Delta, c ; 1-x\right)=\sum_{\Delta^{\prime}} \mathcal{M}_{\Delta}^{\Delta^{\prime}} B\left(\Delta_{1}, \Delta_{2}, \Delta_{3}, \Delta_{4} \mid \Delta^{\prime}, c ; x\right) .
$$


Далее мы опускаем параметры $\Delta_{1}, \Delta_{2}, \Delta_{3}, \Delta_{4}, c$ в обозначении конформного блока, если это не может привести к недоразумению.

Разложение конформного блока (3.1) выводится при помощи ОР

$$
\begin{aligned}
& V_{\Delta_{1} \Delta_{2}}\left(x_{1}, \bar{x}_{1}\right) V_{\Delta_{2}, \bar{\Delta}_{2}}\left(x_{2}, \bar{x}_{2}\right)=\sum_{\Delta} C_{12}^{\Delta, \bar{\Delta}}\left(x_{1}-x_{2}\right)^{\Delta-\Delta_{1}-\Delta_{2}}\left(\bar{x}_{1}-\bar{x}_{2}\right)^{\bar{\Delta}-\bar{\Delta}_{1}-\bar{\Delta}_{2}} \times \\
& \times\left(\sum_{Y, \bar{Y}}\left(x_{1}-x_{2}\right)^{|Y|}\left(\bar{x}_{1}-\bar{x}_{2}\right)^{|\bar{Y}|} \beta_{Y}\left(\Delta \mid \Delta_{1}, \Delta_{2}\right) \bar{\beta}_{\bar{Y}}\left(\bar{\Delta} \mid \bar{\Delta}_{1}, \bar{\Delta}_{2}\right) \hat{L}_{-Y} \hat{\bar{L}}_{-\bar{Y}} V_{\Delta_{2}, \bar{\Delta}_{2}}\left(x_{2}, \bar{x}_{2}\right)\right),
\end{aligned}
$$

где в скобках в правой части представлена сумма по потомкам, которые нумеруются диаграммами Юнга $(Y, \bar{Y})$ всех размеров $|Y|,|\bar{Y}|$. Для простоты мы рассматриваем только ОР примарных полей $V_{1}$ и $V_{2}$ : это возможно, если ограничиться четырехточечными конформными блоками, что мы в действительности и делаем в настоящей работе.

$\mathrm{C}$ помощью ОР можно спроецировать произведения $V_{1}\left(x_{1}\right) V_{2}\left(x_{2}\right)$ и $V_{3}\left(x_{3}\right) V_{4}\left(x_{4}\right)$ на модули Верма, заданные соответственно как $V_{\Delta, \bar{\Delta}}$ и $V_{\Delta^{\prime}, \bar{\Delta}^{\prime}}$, и, поскольку парная корреляционная функция любых полей не равна нулю тогда и только тогда, когда оба поля имеют одну и ту же конформную размерность, мы окончательно приходим к формуле (3.1), и конформный блок представляет киральную часть корреляционной функции с фиксированной промежуточной размерностью. Он может быть полностью определен в рамках киральной алгебры [51].

В терминах киральной алгебры мы определяем конформный блок через киральный коррелятор:

$$
B_{\Delta}(x)=\left\langle\left. V_{\Delta_{1}}\left(x_{1}\right) V_{\Delta_{2}}\left(x_{2}\right)\right|_{\Delta} V_{\Delta}\left(x_{3}\right) V_{\Delta\left(x_{4}\right)}\right\rangle ;
$$

здесь и далее мы обозначаем соответствующее киральное примарное поле той же буквой $V$. Киральное ОР выглядит как

$$
V_{\Delta_{1}}\left(x_{1}\right) V_{\Delta_{2}}\left(x_{2}\right) \stackrel{\Delta}{\longrightarrow}\left(x_{1}-x_{2}\right)^{\Delta-\Delta_{1}-\Delta_{2}}\left(\sum_{Y}\left(x_{1}-x_{2}\right)^{|Y|} \beta_{Y}\left(\Delta \mid \Delta_{1}, \Delta_{2}\right) \hat{L}_{-Y} V_{\Delta}\left(x_{1}\right)\right) .
$$

Коэффициенты $\beta_{Y}$ легко связать с трехточечными функциями: достаточно рассмотреть трехточечную функцию $\Gamma_{Y}\left(\Delta_{1}, \Delta_{2} \mid \Delta\right)=\left\langle V_{\Delta_{1}} V_{\Delta_{2}} \mid \hat{L}_{-Y} V_{\Delta}\right\rangle$ и использовать соотношение (3.5). Тогда мы непосредственно получаем выражение для трехточечной функции:

$$
\Gamma_{Y}\left(\Delta_{1}, \Delta_{2} \mid \Delta\right)=\sum_{Y^{\prime}} T_{Y, Y^{\prime}}(\Delta) \beta_{Y^{\prime}}\left(\Delta \mid \Delta_{1}, \Delta_{2}\right)
$$

с матрицей Шаповалова

$$
T_{Y, Y^{\prime}}(\Delta)=\left\langle\hat{L}_{-Y} V_{\Delta} \mid \hat{L}_{-Y^{\prime}} V_{\Delta}\right\rangle
$$

Заметим, что выделение единственного члена в сумме по $\Delta$ может привести к потере ассоциативности произведения, которая в простейших примерах гарантирована структурными константами $C_{12}^{\Delta, \bar{\Delta}}$. Для многих моделей (когда в спектре имеются вырожденные представления алгебры Вирасоро) эти константы обращаются в ноль для большинства значений $\Delta, \bar{\Delta}$. Однако в этих случаях часто достаточно просто 
наложить на $\Delta$ правила отбора. В любом случае для четырехточечного конформного блока можно избежать использования ассоциативности. На данной стадии мы получаем

$$
\begin{aligned}
B_{\Delta}\left(\Delta_{i} \mid x\right) & =\sum_{Y, Y^{\prime}} x_{12}^{|Y|} x_{34}^{\left|Y^{\prime}\right|} \beta_{Y}\left(\Delta \mid \Delta_{1}, \Delta_{2}\right) \beta_{Y^{\prime}}\left(\Delta \mid \Delta_{3}, \Delta_{4}\right)\left\langle\hat{L}_{-Y} V_{\Delta} \mid \hat{L}_{-Y^{\prime}} V_{\Delta}\right\rangle= \\
& =\sum_{Y, Y^{\prime}} \Gamma_{Y}\left(\Delta_{1}, \Delta_{2} \mid \Delta\right) T_{Y, Y^{\prime}}^{-1} \Gamma_{Y^{\prime}}\left(\Delta_{3}, \Delta_{4} \mid \Delta\right) .
\end{aligned}
$$

Наконец, для того чтобы вычислить трехточечные функции, нужно использовать следующие свойства киральной алгебры.

- Согласованность алгебры Вирасоро со скалярным произведением $\langle\cdot\rangle$ :

$$
\left\langle\hat{L}_{Y} V \mid V^{\prime}\right\rangle=\left\langle V \mid \hat{L}_{-Y} V^{\prime}\right\rangle
$$

для произвольных операторов $V$ и $V^{\prime}$ (не обязательно примарных). Мы нормируем примарные операторы так, что $\left\langle V_{\Delta} \mid V_{\Delta}\right\rangle=1$. На самом деле само это тождество подразумевает, что скалярное произведение в (3.7) равно матрице Шаповалова,

$$
\left\langle\hat{L}_{-Y} V_{\Delta} \mid \hat{L}_{-Y^{\prime}} V_{\Delta}\right\rangle=\left\langle V_{\Delta} \mid \hat{L}_{Y} \hat{L}_{-Y^{\prime}} V_{\Delta}\right\rangle,
$$

в частности, оно исчезает для диаграмм Юнга разных размеров $|Y| \neq|\bar{Y}|$, что важно для проективной инвариантности коррелятора, т. е. для того, чтобы четыре координаты $x_{1}, \ldots, x_{4}$ собирались в одно двойное отношение $x=\frac{\left(x_{2}-x_{1}\right)\left(x_{3}-x_{4}\right)}{\left(x_{3}-x_{1}\right)\left(x_{2}-x_{4}\right)}$. Это позволяет положить $x_{1}=0, x_{2}=x, x_{3}=1, x_{4}=\infty$.

- Копроизведение в алгебре Вирасоро [51], которое является прямым следствием тождеств Уорда [1]:

$$
\hat{L}_{n}\left(V_{1}(0) V_{2}(x)\right)=\left(\sum_{k=0}^{\infty} x^{n+1-k}\left(\begin{array}{c}
n+1 \\
k
\end{array}\right) \hat{L}_{k-1} V_{1}(0)\right) V_{2}(x)+V_{1}(0) \hat{L}_{n} V_{2}(x) .
$$

Применяя эти постулаты, мы получаем [1], [52], [53], что для $Y=\left\{y_{1} \geqslant y_{2} \geqslant \ldots\right\}$ справедливо равенство

$$
\Gamma_{Y}\left(\Delta_{1}, \Delta_{2} \mid \Delta\right)=\prod_{i}\left(\Delta+y_{i} \Delta_{2}-\Delta_{1}-\sum_{j<i} y_{j}\right) .
$$

3.2. Векторы-цепочки. Альтернативно можно использовать очень эффективное представление $B_{k}$ в (1.1) в виде скалярного произведения составляющих выделенных векторов-цепочек [1],

$$
B_{k}=\left\langle k, \Delta_{3}, \Delta_{4} \mid k, \Delta_{1}, \Delta_{2}\right\rangle,
$$

которые зависят также от $\Delta$ и $c$ и удовлетворяют простым определяющим рекуррентным соотношениям

$$
\begin{aligned}
& \hat{L}_{n}\left|k, \Delta_{1}, \Delta_{2}\right\rangle=\left(k-n+\Delta+n \Delta_{2}-\Delta_{1}\right)\left|k-n, \Delta_{1}, \Delta_{2}\right\rangle, \quad 0<n \leqslant k, \\
& \hat{L}_{0}\left|k, \Delta_{1}, \Delta_{2}\right\rangle=(k+\Delta)\left|k, \Delta_{1}, \Delta_{2}\right\rangle .
\end{aligned}
$$


Возможность для данных рекуррентных соотношений однозначно задать цепочку таких состояний в модуле Верма является важной чертой алгебры Вирасоро, которая имеет место также для алгебры $\widehat{U(1)}$, но не для старших алгебр $W_{N}$, начиная с $W_{3}$. В действительности векторы-цепочки по сути есть не что иное, как проекция ОР двух примарных полей с конформными размерностями $\Delta_{1}, \Delta_{2}$ на третье поле с размерностью $\Delta$,

$$
\begin{gathered}
\widehat{V}_{\Delta_{1}}(0) \widehat{V}_{\Delta_{2}}(x) \stackrel{\Delta}{\longrightarrow} x^{\Delta-\Delta_{1}-\Delta_{2}} \sum_{Y} x^{|Y|} \beta_{Y}\left(\Delta \mid \Delta_{1}, \Delta_{2}\right) \hat{L}_{-Y} \widehat{V}_{\Delta}(0), \\
\left|k, \Delta_{1}, \Delta_{2}\right\rangle=\sum_{|Y|=k} \beta_{Y}\left(\Delta \mid \Delta_{1}, \Delta_{2}\right) \hat{L}_{-Y} \widehat{V}_{\Delta}(0),
\end{gathered}
$$

T. e.

$$
B_{k}=\sum_{\left|Y_{1}\right|=\left|Y_{2}\right|=k} \beta_{Y}\left(\Delta \mid \Delta_{1}, \Delta_{2}\right) \beta_{Y}\left(\Delta \mid \Delta_{3}, \Delta_{4}\right)\left\langle\widehat{V}_{\Delta} \hat{L}_{Y_{1}} \mid \hat{L}_{-Y_{2}} \widehat{V}_{\Delta}(0)\right\rangle
$$

Важность векторов-цепочек становится особенно ясна в рамках гипотезы АГТ, поскольку проекторы векторов-цепочек для произведения алгебр Вирасоро и Гейзенберга на особые состояния в модуле Верма, ассоциированные с обобщенными полиномами Джека, воспроизводят функции Некрасова

$$
N_{Y_{1}, Y_{2}}=\left\langle k, \Delta_{3}, \Delta_{4} \mid J_{Y_{1}, Y_{2}}\right\rangle\left\langle J_{Y_{1}, Y_{2}} \mid k, \Delta_{1}, \Delta_{2}\right\rangle
$$

(здесь пара диаграмм Юнга возникает из-за произведения алгебр Вирасоро и Гейзенберга), и у них имеются дополнительные полюсы, которых нет в скалярных произведениях (3.13). Разные реализации этой идеи можно найти в работах $[19]^{2)}$.

3.3. Явные выражения. В результате мы получаем для первых нескольких коэффициентов $B_{k}$ в разложении

$$
B(x)=1+\sum_{k=1}^{\infty} B_{k} x^{k}
$$

следующие явные выражения:

$$
\begin{aligned}
B_{1}= & \frac{\left(\Delta+\Delta_{2}-\Delta_{1}\right)\left(\Delta+\Delta_{3}-\Delta_{4}\right)}{2 \Delta} \\
\mathcal{B}_{\Delta}^{(2)}= & \frac{\left(\Delta+\Delta_{2}-\Delta_{1}\right)\left(\Delta+\Delta_{2}-\Delta_{1}+1\right)\left(\Delta+\Delta_{3}-\Delta_{4}\right)\left(\Delta+\Delta_{3}-\Delta_{4}+1\right)}{4 \Delta(2 \Delta+1)}+ \\
& +\frac{\left(\Delta_{1}+\Delta_{2}\right)(2 \Delta+1)+\Delta(\Delta-1)-3\left(\Delta_{2}-\Delta_{1}\right)^{2}}{2(2 \Delta+1)[2 \Delta(8 \Delta-5)+(2 \Delta+1) c]} \times \\
& \times\left[\left(\Delta_{3}+\Delta_{4}\right)(2 \Delta+1)+\Delta(\Delta-1)-3\left(\Delta_{3}-\Delta_{4}\right)^{2}\right],
\end{aligned}
$$

${ }^{2)} \mathrm{K}$ примеру, на первом уровне $\left|J_{0,1}\right\rangle=\left(\hat{L}_{-1}+(Q+2 a) \hat{a}_{-1}\right)|a\rangle$ и $\left|J_{1,0}\right\rangle=\left(\hat{L}_{-1}+(Q-2 a) \hat{a}_{-1}\right)|a\rangle$, T. e.

$$
\hat{L}_{-1}|a\rangle=-\frac{1}{4 a}\left((Q-2 a)\left|J_{0,1}\right\rangle-(Q+2 a)\left|J_{1,0}\right\rangle\right), \quad \hat{a}_{-1}|a\rangle=\frac{1}{4 a}\left(\left|J_{0,1}\right\rangle-\left|J_{1,0}\right\rangle\right) .
$$


Знаменатели в этих выражениях,

$$
\begin{aligned}
& K_{2}=4 \Delta\left(16 \Delta^{2}-10 \Delta+2 c \Delta+c\right), \\
& K_{3}=6\left(3 \Delta^{2}+c \Delta-7 \Delta+c+2\right) K_{2}, \\
& K_{4}=4(8 \Delta+c-1)\left(16 \Delta^{2}-82 \Delta+10 c \Delta+15 c+66\right) K_{3}, \ldots,
\end{aligned}
$$

при общих значениях $c$ имеют только простые нули, которые совпадают с нулями определителей Каца $K D_{n}$ (т. е. определителей матрицы Шаповалова), хотя последние имеют иногда неединичные кратности. Эти дополнительные нули (с учетом кратности) детерминанта Каца компенсируются нулями числителей в выражениях для четырехточечных конформных блоков, т. е.

$$
K D_{3}=2 \Delta \cdot K_{3}, \quad K D_{4}=4 \Delta^{2}\left(16 \Delta^{2}-10 \Delta+2 c \Delta+c\right) K_{4} .
$$

Поскольку это обращение в ноль имеет место в произвольной точке пространства модулей $\mathcal{M}$ (т. е. для произвольных размерностей и центрального заряда), эти нули не играют никакой роли в нашем дальнейшем рассмотрении, и мы иногда называем редуцированные величины (3.21) детерминантами Каца, предполагая, что это не должно привести к непониманию.

3.4. Коэффициенты $B_{k}$ из представления Доценко-Фатеева [17]. Сделаем замену переменных

$$
\Delta_{\mu}=\alpha_{\mu}\left(\alpha_{\mu}-Q\right), \quad c=1-6 Q^{2}, \quad Q=b-\frac{1}{b},
$$

где $\alpha_{i}$ удовлетворяют условиям

$$
\alpha-\alpha_{1}-\alpha_{2}=b N_{1}, \quad Q-\alpha-\alpha_{3}-\alpha_{4}=b N_{2} .
$$

На самом деле в (3.24) можно выбрать $Q-\alpha_{i}$ вместо любой $\alpha_{i}$, поскольку теория симметрична относительно этой операции (в частности, равенство (3.23) остается неизменным при этом преобразовании).

При такой замене переменных коэффициенты $B_{k}\left(\Delta_{1}, \ldots, \Delta_{4}, \Delta, c\right)$ превращаются в рациональные функции $J_{k}\left(\alpha_{1}, \alpha_{2}+\alpha_{3}, b, N_{1}, N_{2}\right)$, т. е. $B_{k}=J_{k}$, которые при целых неотрицательных значениях $N_{1}$ и $N_{2}$ совпадают со значениями интегралов Сельберга-Каделла [54], взятых $N_{1}$ раз от 0 до $x$ и $N_{2}$ раз от 0 до 1 . Можно интерпретировать этот факт [17], [55] как представление конформных блоков типа Доценко-Фатеева [46] в виде конформной матричной модели [56], [16]. Более подробно,

$$
\begin{gathered}
J_{k}=\frac{Z(v)}{Z(0)}, \\
Z(v)=\int \prod_{a<a^{\prime}}\left(v_{a}-v_{a^{\prime}}\right)^{2 b^{2}} \prod_{a} v_{a}^{2 \alpha_{1} b}\left(x-v_{a}\right)^{2 \alpha_{2} b}\left(1-v_{a}\right)^{2 \alpha_{3} b} d v_{a},
\end{gathered}
$$

и $N_{1}$ раз интегрирование здесь идет от 0 до $x$, в то время как $N_{2}$ раз интегрирование идет от 0 до 1. Заметим, что интегралы не обязательно берутся по замкнутым контурам: для иррациональных произведений $\alpha_{i} \alpha_{j}$ их не так легко определить. Иными 
словами, следуя работам [17], мы определяем интегралы так же, как определен архетипический интеграл для функции $B$ :

$$
\int_{0}^{1} z^{a-1}(1-z)^{b-1} d z=\frac{\Gamma(a) \Gamma(b)}{\Gamma(a+b)} .
$$

Новым здесь является аналитическое продолжение по $N_{1}$ и $N_{2}$ : поскольку $B_{k}$ суть рациональные функции этих переменных, продолжение непосредственно и однозначно.

Для этих формул можно напрямую получить их q-деформацию [21], [22], и такое обобщение связано с пятимерными калибровочными теориями, с $q$-алгебрами Вирасоро, с полиномами Макдональда и с двойными аффинными алгебрами Гекке.

3.5. Общая структура коэффициентов $B_{k}$. Полюсы коэффициентов $B_{k}$ (нули Каца) имеют очень простой вид в параметризации Доценко-Фатеева: они расположены в точках

$$
\alpha_{m, n}=\frac{1}{2}\left(\frac{n-1}{b}-(m-1) b\right), \quad m, n \in \mathbb{Z}, \quad m, n \neq 0, \quad m n \leqslant k .
$$

Отсюда следует, что если полюс появляется в некотором коэффициенте $B_{k}$, то он также появляется во всех остальных $B_{l}$ с $l>k$. Такая сингулярность конформного блока на уровне $k$ может быть убрана, если мы подходящим образом выберем внешние размерности $\Delta_{i}$ так, чтобы теория все еще имела смысл. Достаточно удивительно, что если полюс исчезает каким-либо образом на уровне $k$, он одновременно исчезает на всех старших уровнях. Иными словами, не только нули Каца, но также и нули числителей имеют вложенную структуру. В следующих разделах мы изучим это явление в деталях, в частности мы увидим, что в конформных теориях с рациональными значениями центрального заряда возникают кратные нули Каца, которые иногда все еще сокращаются с кратными нулями в числителе, а иногда - нет.

Нашей целью в дальнейшем является поиск непертурбативных параметров в конформных блоках, т. е. мы ищем конформные блоки, которые представляют собой неоднозначно определенные функции переменной $x$ при данных $\Delta_{i}, \Delta, c$. Один непосредственный пример дает точка $\Delta_{i}=1 / 16, \Delta=1 / 4, c=1$ (см. раздел 4): это случай модели Ашкина-Теллера, решенный Ал. Замолодчиковым [32]. С другой стороны, в той же точке имеется другой конформный блок, задающийся эллиптическим интегралом, который получается из формул, приведенных в работах [17]. Существование двух разных решений может подразумевать, что у нас имеется необходимый непертурбативный параметр. Однако, как мы объясним позже в разделе 5, это не так: неоднозначность возникает вследствие возможности подхода к сингулярности по разным направлениям, поскольку модель Ашкина-Теллера находится в пространстве модулей точно в нуле Каца.

Другая возможность искать непертурбативный параметр могла бы появиться в случаях, когда некоторые из внешних размерностей отвечают вырожденному вектору. Если этот вектор вырожден на уровне $k$ и промежуточная размерность выбрана специальным образом, то конформный блок удовлетворяет дифференциальному уравнению порядка $k$ по $x$. Это уравнение имеет, вообще говоря, $k$ независимых решений, и, следовательно, полный ответ должен являться их линейной комбинацией, зависящей от $k-1$ констант. Однако эти константы не могут быть ассоциированы 
с непертурбативной неоднозначностью в определении конформного блока, поскольку каждое из этих $k$ решений отвечает в точности одному конформному блоку, фиксированному соответствующей асимптотикой! Более того, модулярное преобразование $x \rightarrow 1-x$ преобразует любой из этих конформных блоков через остающиеся $k-1$ блоков.

На самом деле отсутствие непертурбативного параметра во втором случае не удивительно: имеется, как мы уже упоминали, вера в то, что четырехточечный сферический конформный блок как функция от $x$ является функцией, разветвленной над тремя точками 0,1 и $\infty$ без существенных особенностей. Следовательно, ряды разложения по $x$ имеют конечные радиусы сходимости и в этом смысле отличны, скажем, от (2.1). Однако разложение по $x$ сходится неравномерно по другим параметрам $\left(\Delta_{i}, \Delta, c\right)$, и это делает полную функцию всех переменных весьма изысканной, что мы наблюдаем в случае Ашкина-Теллера.

\section{4. НЕПЕРТУРБАТИВНЫЕ ПАРАМЕТРЫ. ПЕРВАЯ ПОПЫТКА: МОДЕЛЬ АШКИНА-ТЕЛЛЕРА}

4.1. Эллиптические интегралы. Заметим, что (3.25) является интегральным представлением полного конформного блока $\mathcal{B}(x)$, а не индивидуальных коэффициентов $J_{k}=B_{k}$ его разложения по $x$. Естественной идеей могло бы быть использование такого представления как очевидного кандидата на непертурбативное определение.

Конечно, эта идея поднимает ряд интересных вопросов, особенно об аналитическом продолжении по $N$ и ассоциированных непертурбативных зависимостях от внутренних размерностей наподобие $\Delta$. Это могло бы открыть дорогу к изучению непертурбативных модулярных ядер [39]-[41], [57], [58] (которые пертурбативно являются просто преобразованиями Фурье [59]). Однако, до того как углубляться в этом направлении, имеет смысл взглянуть на данный подход в более однозначной ситуации: при натуральных (положительных целых) значениях $N_{1}$ и $N_{2}$. Лучше всего начать с простейшего случая $c=1$, т.е. с $b=1$. В этом случае имеется решение (3.24) при $N_{1}=1, N_{2}=0$. Даже после этого возможна дальнейшая конкретизация: специальный выбор внешних размерностей, когда интеграл становится эллиптическим.

$\mathrm{K}$ примеру, при $\alpha_{i}=-1 / 4$ интеграл (3.25) превращается просто в обыкновенный эллиптический интеграл

$$
K(x)=\int \frac{d z}{y(z)}, \quad y^{2}(z)=z(1-z)(z-x),
$$

который можно элементарно проанализировать как пертурбативно, так и непертурбативно:

$$
\int_{0} \frac{d z}{y(z)}=\sum c_{k}^{2} x^{k}=1+\frac{1}{4} x+\frac{9}{64} x^{2}+\cdots
$$

и

$$
\begin{aligned}
\mathcal{B}^{\text {ell }}(x)= & (1-x)^{1 / 8} K(x)=1+\frac{1}{8} x+\frac{7}{128} x^{2}+\frac{33}{1024} x^{3}+\frac{713}{32768} x^{4}+\frac{4165}{262144} x^{5}+ \\
& +\frac{51205}{4194304} x^{6}+\frac{326255}{33554432} x^{7}+\frac{17078585}{2147483648} x^{8}+\frac{114071265}{17179869184} x^{9}+\cdots
\end{aligned}
$$


Команда MAPLE, которая генерирует эту формулу, имеет вид

$$
\operatorname{series}\left((1-x)^{\sim}(1 / 8) * \operatorname{EllipticK}(\operatorname{sqrt}(x)) / P i * 2, x, 10\right) .
$$

Лучший метод непертурбативного анализа интегралов - использовать уравнения Пикара-Фукса для

$$
\Pi=\oint_{C} \frac{d z}{y(z)}=\oint_{C} \frac{d z}{\sqrt{z(1-z)(z-x)}}
$$

вдоль любого замкнутого контура $C$ :

$$
\left(x(1-x) \frac{\partial^{2}}{\partial x^{2}}+(1-2 x) \frac{\partial}{\partial x}+\frac{1}{4}\right) \Pi=0 .
$$

Это уравнение имеет два решения: решение с асимптотикой $K(x) \rightarrow 1$ при малых $x$ и решение с $K^{\prime}(x) \rightarrow \ln x$ при малых $x$ [60]. Нас, конечно, интересует первый случай.

4.2. Формула Замолодчикова. В работе [28] Ал. Замолодчиков предложил замечательную формулу для непертурбативного конформного блока на срезе, где $\Delta_{i}=1 / 16, c=1$ :

$$
\mathcal{B}_{\Delta}^{\operatorname{Zam}}(x)=\left.\mathcal{B}_{\Delta}\left(\Delta_{i}, c \mid x\right)\right|_{\Delta_{i}=1 / 16, c=1}=(1-x)^{-1 / 8} \frac{(16 q / x)^{\Delta}}{\theta_{00}(q)},
$$

где соотношение между $x$, рассматриваемым как точка ветвления, и эллиптическим параметром $q=e^{i \pi \tau}$ дается формулой

$$
x=\frac{\theta_{10}^{4}}{\theta_{00}^{4}}=16 q \frac{\left(1+q^{2}+q^{6}+\cdots\right)^{4}}{\left(1+2 q+2 q^{4}+\cdots\right)^{4}}=16 q-128 q^{2}+\cdots
$$

и тета-константы определяются как

$$
\begin{aligned}
& \theta_{00}(q)=\theta_{00}(0 \mid \tau)=1+2 \sum_{n=1}^{\infty} q^{n^{2}}=\prod_{m=1}^{\infty}\left(1-q^{2 m}\right)\left(1+q^{2 m-1}\right)^{2}, \\
& \theta_{01}(q)=\theta_{01}(0 \mid \tau)=1+2 \sum_{n=1}^{\infty}(-1)^{n} q^{n^{2}}=\prod_{m=1}^{\infty}\left(1-q^{2 m}\right)\left(1-q^{2 m-1}\right)^{2}, \\
& \theta_{10}(q)=\theta_{10}(0 \mid \tau)=2 q^{1 / 4} \sum_{n=0} q^{n(n+1)}=2 q^{1 / 4} \prod_{m=1}^{\infty}\left(1-q^{2 m}\right)\left(1+q^{2 m}\right)^{2}, \\
& \theta_{11}^{\prime}(q)=\theta_{00} \theta_{01} \theta_{10}=2 q^{1 / 4} \prod_{m=1}^{\infty}\left(1-q^{2 m}\right)^{3}=\eta^{3}(q) .
\end{aligned}
$$

Равенство (4.7) связывает модулярные преобразования переменной $x$, генерируемые преобразованиями $x \rightarrow 1-x$ и $x \rightarrow-1 / x+1$, с модулярными преобразованиями тета-констант, которые порождаются соотношениями

$$
\theta_{\epsilon, \delta}(0 \mid-1 / \tau)=(-i)^{\epsilon \delta} \sqrt{-i \tau} \theta_{\delta, \epsilon}(0 \mid \tau), \quad \theta_{\epsilon, \delta}(0 \mid \tau+1)=e^{\pi i / 4 \epsilon} \theta_{\epsilon, \delta+1-\epsilon}(0 \mid \tau),
$$

где характеристики $\epsilon$ и $\delta$ тета-функций следует понимать как взятые по модулю 2. Ключевую роль в этом соотношении играет тождество Римана

$$
\theta_{00}^{4}(0 \mid \tau)=\theta_{01}^{4}(0 \mid \tau)+\theta_{10}^{4}(0 \mid \tau) .
$$


В точке Ашкина-Теллера $\mu_{\text {Ат }}$, где дополнительно $\Delta=1 / 4$,

$$
(1-x)^{1 / 8} \mathcal{B}_{1 / 4}^{\mathrm{Zam}}=\frac{(16 q / x)^{1 / 4}}{\theta_{00}}=\frac{2 q^{1 / 4}}{\theta_{10}}=\frac{1}{1+q^{2}+q^{6}+\cdots},
$$

и пертурбативное разложение по степеням $x$ имеет вид

$$
\begin{aligned}
B_{1 / 4}^{\operatorname{Zam}}(x)= & \left(1+\frac{1}{8} x+\frac{9}{128} x^{2}+\cdots\right)\left(1-\frac{1}{256} x^{2}+\cdots\right)= \\
= & 1+\frac{1}{8} x+\frac{17}{256} x^{2}+\frac{93}{2048} x^{3}+\frac{2269}{65536} x^{4}+\frac{14705}{524288} x^{5}+\frac{198109}{8388608} x^{6}+ \\
& +\frac{1370655}{67108864} x^{7}+\frac{77366631}{4294967296} x^{8}+\frac{554104463}{34359738368} x^{9}+\cdots
\end{aligned}
$$

Команды MAPLE, которые генерируют эту формулу, таковы:

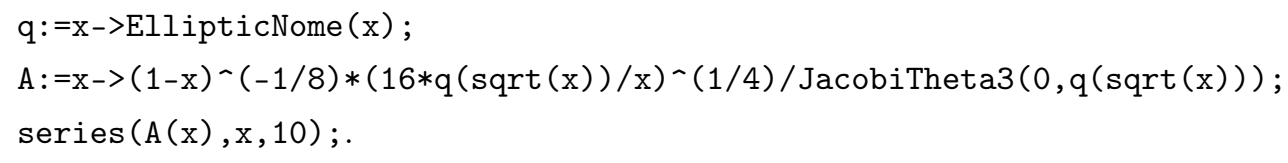

\section{3. Пересечение физического и эллиптического срезов.}

4.3.1. Проблема. Теперь мы можем сравнить два выражения (4.3) и (4.10). Оба соответствуют точке Ашкина-Теллера $\mu_{\text {Ат }}$, для которой

$$
\Delta_{i}=\frac{1}{16}, \quad \Delta=\frac{1}{4}, \quad c=1,
$$

на пространстве модулей $\mathcal{M}$, оба являются разложениями хорошо определенных и хорошо известных функций переменной $x$, но отличаются уже во вторых членах разложений по $x$ ! Что это означает? Как мы можем получить два разных разложения для $B_{2}$ в данной точке?

Причина в том, что $\mu_{\text {Ат }}$ в действительности лежит на дивизоре коэффициента $B_{2}$. Чтобы получить второй коэффициент разложения по $x$ из (3.20), нужно разрешить особенность $0 / 0$, и это разрешение неоднозначно. Конечно, это обычная ситуация для функции многих переменных (даже рациональной, подобной $B_{k}$ ), тем не менее это приводит к дополнительным неоднозначностям в конформном блоке: в нулях Каца он определен конформными размерностями неединственным образом.

Наша непосредственная цель в этой ситуации - изучить окрестность точки $\mu_{\mathrm{Aт}}$ и увидеть, как два выражения (4.3) и (4.10) возникают из единственной формулы (3.20). На следующем шаге мы должны будем посмотреть на другие коэффициенты $B_{k}$ и на другие интересные точки на дивизоре.

4.3.2. Коэффициент $B_{2}$ в окрестности точки $\mu_{\text {Ат. }}$ Итак, мы подставляем

$$
\Delta_{i}=\frac{1}{16}+\epsilon \delta_{i}, \quad \Delta=\frac{1}{4}+\epsilon \delta, \quad c=1+\epsilon \sigma
$$

в выражения (3.19), (3.20) для коэффициентов разложения по $x$ конформного блока и смотрим, что происходит при малых значениях $\epsilon$. 
Для $B_{1}$ мы не получаем ничего интересного:

$$
B_{1}^{\mathrm{AT}}=\frac{1}{8}+O(\epsilon)
$$

и это в точности то, что требуется как в (4.3), так и в (4.10).

Однако для $B_{2}$ ситуация становится гораздо более интересной:

$$
B_{2}^{\mathrm{AT}}=\frac{(2 / 256) \sigma \epsilon+O\left(\epsilon^{2}\right)}{(3 / 2) \sigma \epsilon+O\left(\epsilon^{2}\right)} \stackrel{?}{=} \frac{25}{384}+O(\epsilon) .
$$

Это другое рациональное число, отличное от чисел как в формуле (4.3), так и в формуле (4.10). Давайте тем не менее посмотрим на следующий порядок по $\epsilon$ :

$$
B_{2}^{\mathrm{AT}}=\frac{(2 / 256) \sigma \epsilon+\rho \epsilon^{2}+O\left(\epsilon^{3}\right)}{(3 / 2) \sigma \epsilon+\left(16 \delta^{2}+8 \sigma \delta\right) \epsilon^{2}+O\left(\epsilon^{3}\right)},
$$

где

$$
\rho=\frac{1}{16}\left(17 \delta^{2}-2 \delta\left(\delta_{1}+\delta_{2}+\delta_{3}+\delta_{4}\right)+12\left(\delta_{1}+\delta_{2}\right)\left(\delta_{3}+\delta_{4}\right)\right)+\frac{15 \sigma}{32}\left(2 \delta-\delta_{1}+\delta_{2}+\delta_{3}-\delta_{4}\right) .
$$

Теперь можно понять, что происходит. В обоих случаях (4.3) и (4.10) мы подходим к точке $\mu_{\mathrm{AT}}$, сохраняя $\sigma=0$. Тогда

$$
\left.B_{2}^{\mathrm{AT}}(c)\right|_{c=1}=\frac{1}{256}\left(17-2 \frac{\delta_{1}+\delta_{2}+\delta_{3}+\delta_{4}}{\delta}+12 \frac{\left(\delta_{1}+\delta_{2}\right)\left(\delta_{3}+\delta_{4}\right)}{\delta^{2}}\right)+O(\epsilon) .
$$

Формула Замолодчикова (4.10) ассоциирована с физическим срезом, когда теория и наблюдаемые (внешние размерности $\Delta_{i}$ ) фиксированы: таким образом, мы имеем $\sigma=0, \delta_{1}=\cdots=\delta_{4}=0$, и только промежуточная размерность может варьироваться. Иными словами, мы можем вообразить, что только $\delta$ отлична от нуля. Когда мы подходим к точке $\mu_{\text {Ат }}$ с этого специального направления, мы получаем

$$
\left.\lim _{\delta \rightarrow 0} B_{2}^{\mathrm{AT}}\left(\Delta_{i}, c\right)\right|_{\Delta_{i}=1 / 16, c=1}=\frac{17}{256},
$$

т. е. мы имеем ответ (4.10).

С эллиптическим интегралом (4.3) ситуация совершенно иная. Что в этом случае фиксировано - так это число интегрирований: $N_{1}=1, N_{2}=0$. Согласно равенствам (3.24) это подразумевает, что мы подходим к точке $\mu_{\text {Aт }}$ с совершенно иного направления, а именно

$$
\sigma=0, \quad \delta+2\left(\delta_{1}+\delta_{2}\right)=0, \quad \delta-2\left(\delta_{3}+\delta_{4}\right)=0 .
$$

Заметим, что равенства (3.24) написаны в терминах параметров $\alpha$, а не размерностей, и при $c=1$ наши $\delta_{i}=\left(\alpha_{i}-\alpha_{i}^{\mathrm{AT}}\right) / 2 \epsilon \sqrt{\Delta_{i}}$, отсюда возникает добавочный коэффициент $2=\sqrt{1 / 4} / \sqrt{1 / 16}$ в (4.19). Тогда формула (4.17) дает при условии (4.19)

$$
\left.\lim _{\delta \rightarrow 0} B_{2}^{\mathrm{AT}}\left(\Delta_{i}, c\right)\right|_{\Delta_{i}=1 / 16, c=1}=\frac{1}{256}\left(17-\frac{12}{2^{2}}\right)=\frac{7}{128},
$$

т. е. в точности то, что нужно для (4.3). Заметим, что условия (4.19) налагают только два ограничения, но этого оказывается достаточно, чтобы обеспечить однозначный предел в (4.17). 
На самом деле рассматривать центральный заряд и размерности на равных основаниях и удобно, и естественно. Если мы вводим параметризацию $c=1-6(b-1 / b)^{2}$ (см. (3.23)) и используем $b=1+\epsilon \eta$ вместо $c=1+\epsilon \sigma$, то отклонения от точки Ашкина-Теллера будут иметь порядок $\epsilon^{2}$ в числителе и в знаменателе. Иными словами,

$$
\sigma=-24 \eta^{2} \epsilon
$$

и именно это разрешение особенности мы используем в оставшейся части данного раздела. В частности, в этой параметризации

$$
B_{2}^{\mathrm{AT}}=\frac{\frac{17}{16} \delta^{2}-\frac{75}{32} \eta^{2}-\frac{1}{8} \delta\left(\delta_{1}+\delta_{2}+\delta_{3}+\delta_{4}\right)+\frac{3}{4}\left(\delta_{1}+\delta_{2}\right)\left(\delta_{3}+\delta_{4}\right)+O(\epsilon)}{16 \delta^{2}-36 \eta^{2}+O(\epsilon)},
$$

где мы опустили общие множители $\epsilon^{2}$ в числителе и в знаменателе.

4.3.3. Другие коэффициенты $B_{k}$ : универсальность и росток конформного блока в точке $\mu_{\text {Ат }}$. Если теперь сделать ту же подстановку в $B_{3}$, снова получится двойной ноль в точке $\mu_{\mathrm{Aт}}$ в числителе и в знаменателе, и мы имеем

$$
B_{3}^{\mathrm{AT}}=\frac{\frac{7533}{1024} \delta^{2}-\frac{32805}{2048} \eta^{2}-\frac{729}{512} \delta\left(\delta_{1}+\delta_{2}+\delta_{3}+\delta_{4}\right)+\frac{2187}{256}\left(\delta_{1}+\delta_{2}\right)\left(\delta_{3}+\delta_{4}\right)+O(\epsilon)}{162 \delta^{2}-\frac{729}{2} \eta^{2}+O(\epsilon)}
$$

Числа могут смотреться ужасно, однако на самом деле они в точности такие же, как в (4.22):

$$
B_{3}^{\mathrm{AT}}=-\frac{15}{512}+\frac{9}{8} B_{2}^{\mathrm{AT}}+O(\epsilon)
$$

Это означает, что нам не нужно делать никакого независимого вычисления в третьем члене в (4.3) и (4.10): взаимосвязь этих двух случаев полностью фиксируется на уровне второго коэффициента.

Действительно, то же свойство выполняется для $B_{4}$ и старших коэффициентов $B_{k}$ :

$$
\begin{gathered}
B_{1}=\frac{1}{8}, \quad B_{2}=\frac{17-r}{256}, \quad B_{3}=\frac{93-9 r}{2^{12}}, \quad B_{4}=\frac{2269-281 r}{2^{16}}, \\
B_{5}=\frac{14705-2125 r}{2^{19}}=\frac{5 \cdot 17}{2^{19}}(173-25 r), \quad \ldots,
\end{gathered}
$$

где

$$
r \equiv \frac{-3 \eta^{2}+8 \delta\left(\delta_{1}+\delta_{2}+\delta_{3}+\delta_{4}\right)-48\left(\delta_{1}+\delta_{2}\right)\left(\delta_{3}+\delta_{4}\right)}{4 \delta^{2}-9 \eta^{2}} .
$$

Естественно предположить, что это остается верным в общем случае:

$$
B_{k}=B_{k}^{\mathrm{Zam}}+\frac{r}{3}\left(B_{k}^{\mathrm{ell}}-B_{k}^{\mathrm{Zam}}\right)
$$

Однако на самом деле это так только для $k<6$. Действительно, на уровне 6 ноль Каца в точке $\Delta=1 / 4$ имеет четвертый порядок: $(n-m)^{2} / 4=1 / 4$, если $n=2$, $m=1$, т. е. на уровне $n m=2$; если $n=3, m=2$, т. е. на уровне $n m=6$ и т. д. Числитель все еще сокращает этот кратный ноль в точке Ашкина-Теллера, однако 
неоднозначность становится отношением двух полиномов четвертого порядка от $\delta_{i}$, $\delta, \eta$. Для $6 \leqslant k<12$ мы имеем

$$
B_{k}=B_{k}^{\text {Zam }}+\frac{r}{3}\left(B_{k}^{\text {ell }}-B_{k}^{\text {Zam }}\right)+\frac{r_{2}}{3 \cdot 2^{11}} C_{k},
$$

где

$$
C_{1}=\cdots=C_{5}=0, \quad C_{6}=1, \quad C_{7}=\frac{25}{8}, \quad C_{8}=\frac{1577}{800} C_{7}, \ldots
$$

и

где

$$
r_{2}=\frac{P_{4}\left(\eta, \delta, \delta_{i}\right)}{\left(4 \delta^{2}-9 \eta^{2}\right)\left(4 \delta^{2}-25 \eta^{2}\right)},
$$

$$
\begin{aligned}
& P_{4}\left(\eta, \delta, \delta_{i}\right)=-60 \eta^{4}+\eta^{2}\left(15 \delta^{2}+86 \delta\left(\delta_{1}+\delta_{2}+\delta_{3}+\delta_{4}\right)+120\left(\delta_{1}+\delta_{2}\right)^{2}+\right. \\
& \left.\quad+120\left(\delta_{3}+\delta_{4}\right)^{2}-660\left(\delta_{1}+\delta_{2}\right)\left(\delta_{3}+\delta_{4}\right)\right)- \\
& \quad-8 \delta^{3}\left(\delta_{1}+\delta_{2}+\delta_{3}+\delta_{4}\right)+48 \delta^{2}\left(3\left(\delta_{1}+\delta_{2}\right)\left(\delta_{3}+\delta_{4}\right)-\left(\delta_{1}+\delta_{2}\right)^{2}-\left(\delta_{3}+\delta_{4}\right)^{2}\right)- \\
& \quad-192 \delta\left(\delta_{1}+\delta_{2}\right)\left(\delta_{3}+\delta_{4}\right)\left(\delta_{1}+\delta_{2}+\delta_{3}+\delta_{4}\right)+960\left(\delta_{1}+\delta_{2}\right)^{2}\left(\delta_{3}+\delta_{4}\right)^{2} .
\end{aligned}
$$

Мораль этой истории состоит в том, что поведение конформного блока в окрестности точки $\mu_{\text {Ат }}$ универсально: оно не зависит от порядка $k$ разложения по $x$. Это открывает возможность предложить формулу для ростка конформного блока в точке $\mu_{\mathrm{Aт}}$ (следующий коэффициент $r_{3}$ возникает на уровне 12 , когда ноль Каца имеет кратность 6):

$$
\begin{aligned}
B(x)= & B^{\text {Zam }}(x)+\frac{r}{3}\left(B^{\text {ell }}(x)-B^{\text {Zam }}(x)\right)+ \\
& +\frac{r_{2}}{3} \frac{x^{6}}{2^{11}}\left(1+\frac{25}{8} x+\frac{1577}{256} x^{2}+\frac{20141}{2048} x^{3}+\frac{911193}{65536} x^{4}+\frac{9549597}{524288} x^{5}+\cdots\right)+ \\
& +x^{12} B_{12}+\cdots+O(\epsilon) .
\end{aligned}
$$

Разложение Замолодчикова соответствует всем внешним $\delta_{i}=0$, в то время как эллиптический локус в окрестности точки Ашкина-Теллера является объединением нескольких гиперплоскостей, причем каждая из них определена двумя условиями:

$$
\sum_{i=1}^{4} \delta_{i}=-\frac{3}{2} \eta, \quad \delta=2\left(\delta_{3}+\delta_{4}\right)+\eta
$$

или

$$
\sum_{i=1}^{4} \delta_{i}=\frac{3}{2} \eta, \quad \delta+2\left(\delta_{3}+\delta_{4}\right)=2 \eta .
$$

4.4. Векторы-цепочки. В качестве альтернативы описанной технике можно предложить анализ локуса сингулярностей в терминах представления коэффициентов $B_{k}$ в (1.1) через векторы-цепочки (см. п. 3.2). К примеру, в точке Ашкина-Теллера лидирующее поведение векторов-цепочек задается формулами

$$
\begin{gathered}
\beta_{2}=-\frac{\zeta}{\xi}+O(\xi), \\
\beta_{11}=\frac{\zeta}{\xi}+O(\xi), \quad \beta_{21}=-\frac{\zeta}{2 \xi}+O(\xi), \quad \beta_{111}=\frac{\zeta}{2 \xi}+O(\xi), \\
\beta_{3}=0,
\end{gathered}
$$


где

$$
\zeta=\frac{\delta-6 \delta_{1}-6 \delta_{2}}{2\left(32 \delta^{2}+3 \eta\right)}
$$

и $\xi$ - расстояние до локуса сингулярностей. Таким образом, векторы-цепочки являются ненулевыми векторами вблизи локуса, но должны иметь нулевую норму в лидирующем порядке, поскольку их норма (которая равна конформному блоку (3.13)) конечна на локусе. Действительно, сингулярности в конформном блоке сокращаются из-за вырожденности матрицы Шаповалова (поскольку локус сингулярностей расположен в нулях детерминанта матрицы Шаповалова):

$$
\left(\sum_{|Y|=k} \operatorname{sing}\left(\beta_{Y}\right)\right)^{2}=0 .
$$

\section{5. ПЕРТУРБАТИВНЫЙ КОНФОРМНЫЙ БЛОК В ОКРЕСТНОСТИ ДИВИЗОРА КАЦА}

5.1. Полюсы коэффициентов $B_{k}$ и их вложенная структура. Мы уже обсуждали в разделе 3 и демонстрировали явно на примере модели Ашкина-Теллера в разделе 4 следующее.

- Если в детерминанте Каца $K_{m}$ появляется ноль, он сохраняется во всех высших детерминтанатах $K_{k}$ с $k \geqslant m$. Детерминанты Каца зависят только от промежуточной размерности $\Delta$ и центрального заряда $c$, и в $\alpha$-параметризации (3.23) нули на самом деле расположены в точках (3.26). В этих точках все коэффициенты $B_{k}$ с $k \geqslant m$ сингулярны.

- Можно, однако, подогнать внешние размерности $\Delta_{i}$ так, что числитель в $B_{m}$ также обращается в ноль. И, как оказывается, знаменатели всех старших $B_{k}$ с $k \geqslant m$ тогда тоже равны нулю.

$\mathrm{K}$ примеру, ноль Каца на третьем уровне находится в точке $\alpha_{1,3}=-b$. Числитель коэффициента $B_{3}$ в этой точке равен

$$
X=\frac{X_{12} X_{34}}{24 b^{5}\left(b^{2}-1\right)\left(3 b^{2}-1\right)\left(4 b^{4}-1\right)},
$$

где

$$
\begin{aligned}
X_{12}= & \left(\alpha_{1}-\alpha_{2}\right)\left(b^{2}-1-b \alpha_{1}-b \alpha_{2}\right)\left(2 b^{2}-1-b \alpha_{1}-b \alpha_{2}\right) \times \\
& \times\left(b+\alpha_{1}-\alpha_{2}\right)\left(b-\alpha_{1}+\alpha_{2}\right)\left(1+b \alpha_{1}+b \alpha_{2}\right) .
\end{aligned}
$$

Числитель коэффициента $B_{4}$ равен

$$
\begin{aligned}
X \cdot & \frac{2 b^{3}+2 b+\alpha_{2}^{2} b-\alpha_{2} b^{2}+\alpha_{2}-\alpha_{1}^{2} b+\alpha_{1} b^{2}-\alpha_{1}}{4 b^{2}\left(b^{2}+1\right)} \times \\
& \times\left(2 b^{3}+2 b+\alpha_{3}^{2} b-\alpha_{3} b^{2}+\alpha_{3}-\alpha_{4}^{2} b+\alpha_{4} b^{2}-\alpha_{4}\right) .
\end{aligned}
$$

Это показывает, что в нуле Каца на уровне $m=3$ ноль числителя коэффициента $B_{m}(X=0)$ остается нулем старших коэффициентов $B_{k}$, например $B_{4}$.

Подчеркнем еще раз, что не только локусы нулей $V\left(K_{k}\right)$ детерминантов Каца обладают вложенной структурой,

$$
V\left(K_{k}\right) \subset V\left(K_{l}\right), \quad \text { или } K_{l} \vdots K_{k} \text { для } l>k,
$$




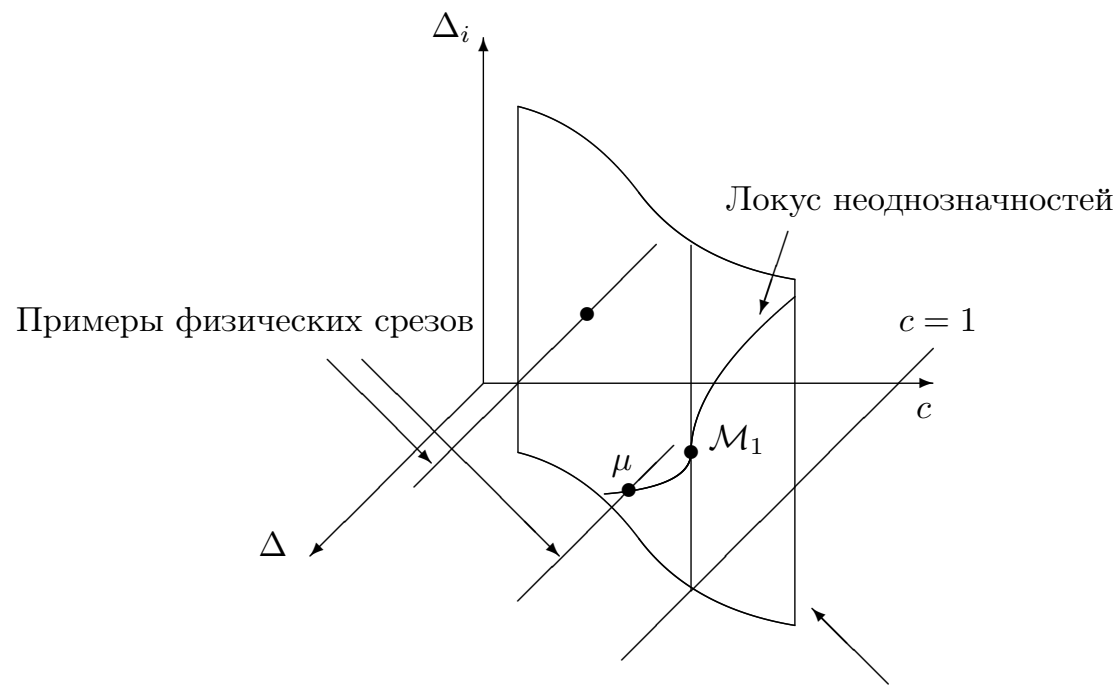

Локус сингулярностей

Рис. 1. Локус неоднозначностей: ноль всех $B_{k}$ с $k \geqslant m$ (гиперповерхность коразмерности два), примеры физических срезов (одномерные гиперповерхности при фиксированных значениях $\Delta_{i}$ и $c$ ) и локус сингулярностей: уровень Каца на уровне $m$ (гиперповерхность коразмерности один).

то же справедливо для числителей коэффициентов $B_{k}$, и это обеспечивается ограничением для $V\left(K_{k}\right)$, а именно для множества $\mathcal{V}_{k}$ нулей коэффициента $B_{k}$ на $V\left(K_{k}\right)$ мы имеем

$$
\mathcal{V}_{k} \subset \mathcal{V}_{l} \quad \text { для } \quad k<l \text {. }
$$

\section{2. Коэффициенты $B_{k}$ в точках пересечения множеств нулей числи-} телей и знаменателей. В общей точке множества $\bigcup_{k} V\left(K_{k}\right)$ коэффициенты конформного блока сингулярны (имеют полюсы), таким образом, это объединение образует локус сингулярностей на пространстве модулей $\mathcal{M}$ и имеет коразмерность один. Однако существует гиперповерхность коразмерности один внутри этого локуса сингулярностей (таким образом, мы имеем коразмерность два в $\mathcal{M}$ ), где числители также обращаются в ноль. Во всех этих точках имеется неоднозначность вида 0/0, причем значения коэффициентов зависят от направления, по которому мы подходим к такой точке в $\mathcal{M}$. Естественно называть эту гиперповерхность коразмерности два локусом неоднозначностей (см. рис. 1). Нашей следующей целью является описание поведения полного конформного блока, а не просто его частных коэффициентов $B_{k}$ в этом локусе.

Предметом нашего внимания в этом разделе будут точки $\mu \in \mathcal{A} \subset \mathcal{M}$, лежащие на локусе неоднозначности $\mathcal{A}$. Суть в том, что большинство хорошо известных примеров конформных моделей, включая модель Ашкина-Теллера и минимальные модели, относятся к этому типу. Как мы уже видели при обсуждении окрестности точки $\mu_{\text {Ат }} \in \mathcal{M}_{1} \subset \mathcal{A} \subset \mathcal{M}$, все коэффициенты $B_{k}$ могут вести себя схожим образом 
вблизи этого локуса, таким образом, их общие свойства полностью наследуются полным конформным блоком.

Как и ранее, в окрестности точки $\mu \in \mathcal{A}, \mu=\left\{\Delta_{i}^{(0)}, \Delta^{(0)}, c^{(0)}\right\}$, мы полагаем

$$
\Delta_{i}=\Delta_{i}^{(0)}+\epsilon \delta_{i}, \quad \Delta=\Delta^{(0)}+\epsilon \delta, \quad c=c^{(0)}+\epsilon \sigma
$$

или $b=b^{(0)}+\epsilon \eta$ с 6 -вектором $\xi_{I}=\left(\delta, \delta_{1}, \ldots, \delta_{4}, \eta\right)$. Оказывается, что

$$
B_{k}=b_{k}^{(0)}+d_{k} \frac{u_{I} \xi_{I}+O\left(\xi^{2}\right)}{v_{I} \xi_{I}+O\left(\xi^{2}\right)},
$$

если ноль простой,

$$
B_{k}=b_{k}^{(0)}+d_{k} \frac{U_{I J} \xi_{I} \xi_{J}+O\left(\xi^{3}\right)}{V_{I J} \xi_{I} \xi_{J}+O\left(\xi^{3}\right)},
$$

если ноль двойной, и т. д. Часто случается (как в предыдущем разделе), что

$$
\text { линейные/квадратичные формы } u, v \text { и } U, V \text { не зависят от } k \text {. }
$$

Заметим, что модифицированная параметризация (4.21) существенна, только если $c^{(0)}=1$, в противном случае $\sigma$ и $\eta$ связаны линейно безо всякого дополнительного подавляющего фактора $\epsilon$.

Таким образом, в критической точке имеются как неоднозначность (предел зависит от выбора направления подхода к точке), так и универсальность, выраженная соотношением (5.5) и условием (5.9).

В остальной части этого раздела мы рассмотрим больше примеров, для которых справедливы (5.5) и (5.9).

5.3. Явление 1: вложенная структура нулей. Пример $c=1$. При $c=1$ все нули детерминантов Каца двойные $\left(\Delta_{0}=\alpha^{2}\right.$ само является полным квадратом):

$$
\begin{aligned}
& K_{2}=4 \Delta(4 \Delta-1)^{2}, \\
& K_{3}=72 \Delta(4 \Delta-1)^{2}(\Delta-1)^{2}, \\
& K_{4}=2304 \Delta^{2}(4 \Delta-1)^{2}(\Delta-1)^{2}(4 \Delta-9)^{2}, \ldots .
\end{aligned}
$$

С числителями коэффициентов $B_{k}$ при $c=1$ не происходит ничего специального. $\mathrm{K}$ примеру, если взять все внешние размерности равными друг другу, $\Delta_{1}=\cdots=$ $\Delta_{4}=\Delta_{\mathrm{e}}$, то числители коэффициентов $B_{2}, B_{3}$ равны соответственно

$$
\begin{aligned}
N B_{2}= & \Delta\left(\Delta-6 \Delta^{2}+9 \Delta^{3}+8 \Delta^{4}+16 \Delta_{\mathrm{e}}^{2} \Delta-8 \Delta_{\mathrm{e}} \Delta+8 \Delta_{\mathrm{e}} \Delta^{2}+8 \Delta_{\mathrm{e}}^{2}\right), \\
N B_{3}= & 3 \Delta(\Delta+2)(\Delta-1)^{2} \times \\
& \times\left(8 \Delta^{4}+19 \Delta^{3}+24 \Delta_{\mathrm{e}} \Delta^{2}-11 \Delta^{2}+48 \Delta_{\mathrm{e}}^{2} \Delta-24 \Delta_{\mathrm{e}} \Delta+2 \Delta+24 \Delta_{\mathrm{e}}^{2}\right) .
\end{aligned}
$$

Дело, однако, значительно меняется, если смотреть на эти числители в нулях Каца. К примеру, при $\Delta=\alpha_{2,1}^{2}=\Delta_{2,1}=\Delta_{1,2}=1 / 4$ (при $c=1$ ) мы имеем

$$
\begin{aligned}
N B_{2}= & \frac{3}{256}\left(1-8\left(\Delta_{1}+\Delta_{2}\right)+16\left(\Delta_{1}^{2}+\Delta_{2}^{2}\right)-32 \Delta_{1} \Delta_{2}\right) \times \\
& \times\left(1-8\left(\Delta_{3}+\Delta_{4}\right)+16\left(\Delta_{3}^{2}+\Delta_{4}^{2}\right)-32 \Delta_{3} \Delta_{4}\right) \equiv \frac{3}{256} n b_{2}, \\
N B_{3}= & -\frac{27}{16384}\left(9+4\left(\Delta_{1}-\Delta_{2}\right)\right)\left(9+4\left(\Delta_{3}-\Delta_{4}\right)\right) n b_{2}, \quad \ldots,
\end{aligned}
$$


т. е. $N B_{k} \sim n b_{2}$ при $k \geqslant 2$. Это показывает, как соотношение (5.5) реализуется в данном случае: любой ноль числителя $N B_{2}$ остается нулем в старших $N B_{k}$.

Посмотрим теперь на окрестность двойного нуля величины $N B_{2}$. В этом случае

$$
\Delta_{1}=\Delta_{2} \pm \sqrt{\Delta_{2}}+\frac{1}{4}, \quad \Delta_{4}=\Delta_{3} \pm \sqrt{\Delta_{3}}+\frac{1}{4}
$$

и мы имеем (выбирая оба знака плюс для определенности)

$$
\begin{aligned}
B_{2}= & \frac{\sqrt{\Delta_{2} \Delta_{3}}\left(4 \sqrt{\Delta_{2} \Delta_{3}}-2 \sqrt{\Delta_{2}}-2 \sqrt{\Delta_{3}}+3\right)}{4}+\frac{\sqrt{\Delta_{2} \Delta_{3}}\left(2 \sqrt{\Delta_{2}}+1\right)\left(2 \sqrt{\Delta_{3}}+1\right)}{4} r \\
B_{3}= & \frac{\sqrt{\Delta_{2} \Delta_{3}}\left(20 \Delta_{2} \Delta_{3}+6 \Delta_{2} \sqrt{\Delta_{3}}+6 \Delta_{3} \sqrt{\Delta_{2}}+4 \Delta_{2}+4 \Delta_{3}+27 \sqrt{\Delta_{2} \Delta_{3}}-6 \sqrt{\Delta_{2}}-6 \sqrt{\Delta_{3}}+8\right)}{18}+ \\
& +\frac{\left(\sqrt{\Delta_{2}}-2\right)\left(\sqrt{\Delta_{3}}-2\right)}{18} \frac{\sqrt{\Delta_{2} \Delta_{3}}\left(2 \sqrt{\Delta_{2}}+1\right)\left(2 \sqrt{\Delta_{3}}+1\right)}{4} r, \quad \ldots,
\end{aligned}
$$

где

$$
r \equiv \frac{3 \eta^{2}-4 \delta\left(\hat{\delta}_{1}+\hat{\delta}_{2}+\hat{\delta}_{3}+\hat{\delta}_{4}\right)-6\left(\hat{\delta}_{1} \hat{\delta}_{3}+\hat{\delta}_{2} \hat{\delta}_{4}\right)+12 \hat{\delta}_{1} \hat{\delta}_{4}+3 \hat{\delta}_{2} \hat{\delta}_{3}}{4 \delta^{2}-9 \eta^{2}}
$$

и мы ввели перемасштабированные величины

$$
\hat{\delta}_{1}=\frac{\delta_{1}}{2 \sqrt{\Delta_{2}}+1}, \quad \hat{\delta}_{2}=\frac{\delta_{2}}{\sqrt{\Delta_{2}}}, \quad \hat{\delta}_{3}=\frac{\delta_{3}}{\sqrt{\Delta_{3}}}, \quad \hat{\delta}_{4}=\frac{\delta_{4}}{2 \sqrt{\Delta_{3}}+1} .
$$

Это показывает, как соотношение (5.9) работает в случае $c=1$.

Подставляя подобным образом $\Delta=\alpha_{3,1}^{2}=\Delta_{3,1}=\Delta_{1,3}=1$ (при $c=1$ ), получаем

$$
\begin{aligned}
N B_{3}= & -18\left(\Delta_{1}-\Delta_{2}\right)\left(1-2\left(\Delta_{1}+\Delta_{2}\right)+\left(\Delta_{1}-\Delta_{2}\right)^{2}\right) \times \\
& \times\left(\Delta_{3}-\Delta_{4}\right)\left(1-2\left(\Delta_{3}+\Delta_{4}\right)+\left(\Delta_{3}-\Delta_{4}\right)^{2}\right), \\
N B_{4}= & 100\left(4-\Delta_{1}+\Delta_{2}\right)\left(4+\Delta_{3}-\Delta_{4}\right) \cdot N B_{3}, \quad \ldots,
\end{aligned}
$$

т. е. $N B_{k} \sim N B_{3}$ при $k \geqslant 3$. Таким образом, мы видим, что любой ноль числителя $N B_{3}$ остается нулем старших $N B_{k}$.

Можно легко проверить, что это остается верным и для других нулей Каца.

\section{4. Явление 2: вложенная структура не всегда усилена в кратных} (нерегулярных) полюсах. Пример $c=7 / 10$ и $c=1 / 2$. При $c=7 / 10$ имеется простой полюс в $\Delta=3 / 2$ на третьем уровне, т. е. в коэффициенте $B_{3}$, в то время как в $B_{4}$ он становится двойным полюсом. Это происходит потому, что при $b=\sqrt{5} / 2$ присутствует добавочное вырождение: $\Delta_{1,3}=\Delta_{4,1}=3 / 2$.

Если все $\Delta_{i}=3 / 2$, числители и коэффициента $B_{3}$, и коэффициента $B_{4}$ содержат только ноль первого порядка, так что $B_{4}$ остается бесконечным, в то время как $B_{3}$ - просто неоднозначным, что не похоже на примеры, рассмотренные нами ранее. К примеру, в модели Ашкина-Теллера неединичная кратность полюса немедленно приводит к неединичной кратности соответствующего нуля числителя конформного блока. Это означает, что при $c=7 / 10$ структурные константы $C_{3 / 2,3 / 2}^{3 / 2}$ должны быть равны нулю (в то время как в модели Ашкина-Теллера в этом нет необходимости). В действительности это налагает ограничения на рациональные конформные теории, поскольку при рациональных значениях центрального заряда в конформном блоке всегда появляются полюсы со старшими кратностями. 
При $c=7 / 10$ проблема возникает в симметричной точке, когда все внешние размерности равны друг другу. Однако подобное явление имеет место и в более простой теории с $c=1 / 2$, хотя и в несимметричной точке. Опишем это несколько более детально.

Если центральный заряд $c=1 / 2$, детерминанты Каца равны

$$
\begin{aligned}
& K_{2}=2 \Delta_{0}\left(16 \Delta_{0}-1\right)\left(2 \Delta_{0}-1\right) \\
& K_{3}=6 \Delta_{0}\left(16 \Delta_{0}-1\right)\left(2 \Delta_{0}-1\right)^{2}\left(3 \Delta_{0}-5\right), \\
& K_{4}=6 \Delta_{0}\left(16 \Delta_{0}-1\right)^{2}\left(2 \Delta_{0}-1\right)^{2}\left(16 \Delta_{0}-21\right)\left(3 \Delta_{0}-5\right)\left(2 \Delta_{0}-7\right), \ldots \ldots
\end{aligned}
$$

Двойные нули в этих формулах возникают вследствие совпадения размерностей при $c=1 / 2$ :

$$
\Delta_{1,3}=\Delta_{2,1}=\frac{1}{2}, \quad \Delta_{2,2}=\Delta_{1,2}=\frac{1}{16}
$$

Однако эти случайно усиленные нули не приводят к дополнительным полюсам в коэффициентах $B_{3}$ и $B_{4}$ : при произвольных значениях четырех внешних размерностей $B_{3} \sim \Delta_{0}-1$, а $B_{4} \sim\left(16 \Delta_{0}-1\right)\left(2 \Delta_{0}-1\right)$, и это гарантирует, что полюсы остаются простыми.

Тем не менее ситуация оказывается не столь тривиальной. Остающийся простой ноль все еще нужно компенсировать в числителе, и это теперь уже нельзя сделать универсальным образом. Действительно, рассмотрим конформный блок теории с $c=1 / 2$ с первыми двумя внешними размерностями, параметризованными каK

$$
\Delta_{1}=\frac{x^{2}-1}{48}+\frac{1}{3}+\frac{x}{6}, \quad \Delta_{2}=\frac{x^{2}-1}{48},
$$

это гарантирует, что ноль в знаменателе $B_{2}$ простой. Однако в $B_{3}$ это не обеспечивает дополнительный ноль вдобавок к фактору $2 \Delta_{0}-1$, который возникает независимо от внешних размерностей. Таким образом, разрушается вложенная структура (5.5), и необходимо налагать дополнительные ограничения, чтобы избежать появления бесконечностей.

5.5. Явление 3: универсальность. Пример $c=1 / 2$. Лидирующее поведение коэффициента $B_{3}$ в окрестности сингулярности задается соотношением

$$
B_{3}=\frac{(x+5)(x-1)(x+2)\left(\Delta_{3}-\Delta_{4}\right)\left(2 \Delta_{3}^{2}-4 \Delta_{3} \Delta_{4}+2 \Delta_{4}^{2}-3 \Delta_{3}-3 \Delta_{4}+1\right)}{3^{4} 2^{3}(3 \sigma-7 \delta)} \frac{1}{\epsilon}+O\left(\epsilon^{0}\right) .
$$

Следовательно, можно либо выбрать специальное значение $x$, либо специальным образом подогнать $\Delta_{3}$ и $\Delta_{4}$.

В первом случае можно выбрать, к примеру, $x=-2$. Тогда вложенная структура восстанавливается - этого условия достаточно, чтобы сократить полюсы в коэффициентах $B_{3}, B_{4}, \ldots$. Однако универсальность условия (5.9) нарушается подобно 
случаю Ашкина-Теллера в предыдущем разделе: конформный блок выглядит как

$$
\begin{aligned}
B_{1}= & \frac{2 \Delta_{3}-\Delta_{4}+1}{4}, \\
B_{2}= & \frac{1}{7 \cdot 2^{5}}\left(36\left(\Delta_{3}^{2}+\Delta_{4}^{2}-\Delta_{3} \Delta_{4}\right)+88 \Delta_{3}-80 \Delta_{4}+31\right)+ \\
& +\frac{1}{7 \cdot 2^{5}}\left(12 \Delta_{3}^{2}-24 \Delta_{3} \Delta_{4}+12 \Delta_{4}^{2}-8 \Delta_{3}-8 \Delta_{4}+1\right) r, \\
B_{k}= & B_{k}^{(0)}+B_{k}^{(1)} r+B_{k}^{(2)} r_{2}, \quad 3 \leqslant k \leqslant 24,
\end{aligned}
$$

поскольку полюс в $\Delta=1 / 2$ становится тройным на уровне $25: \Delta_{(5,5)}=1 / 2$ при $c=1 / 2$. Здесь

$$
r \equiv \frac{\sigma-28 \delta_{1}-28 \delta_{2}}{7 \delta+2 \sigma}, \quad r_{2} \equiv \frac{\delta_{1}-\delta_{2}}{3 \sigma-7 \delta}
$$

Во втором случае для подгонки значений размерностей $\Delta_{3}$ и $\Delta_{4}$ можно использовать ту же параметризацию (5.19),

$$
\Delta_{4}=\frac{y^{2}-1}{48}+\frac{1}{3}+\frac{y}{6}, \quad \Delta_{3}=\frac{y^{2}-1}{48},
$$

так что

$$
B_{3}=\frac{5}{3^{7} 2^{7}} \frac{(x+5)(x-1)(x+2)(y+5)(y-1)(y+2)}{3 \sigma-7 \delta} \frac{1}{\epsilon}+O\left(\epsilon^{0}\right),
$$

и достаточно выбрать $y=1, y=-2$ или $y=-5$. Однако в этом случае двух ограниченных размерностей и одной подогнанной так, чтобы сократить полюс в знаменателе, т. е. в случае однопараметрического подпространства в четырехмерном пространстве переменных $\left(\Delta_{1}, \Delta_{2}, \Delta_{3}, \Delta_{4}\right)$, можно наблюдать новое явление.

5.6. Явление 4: дополнительная универсальность минимальных моделей. Пример модели Изинга. В случае четырех внешних размерностей, параметризованных двумя переменными $(x, y)$, как выше (так что в числителе имеется нужный ноль), конформный блок при, скажем, $x=-2$ равен

$$
\begin{aligned}
B_{1}= & \frac{1-y}{2} \\
B_{2}= & \frac{(y-1)(y-7)}{3 \cdot 2^{6}}, \\
B_{3}= & -\frac{5}{3^{4} 2^{8}}(y-1)(y-7)(y-13)-\frac{5}{3^{4} 2^{6}}(y-1)(y+2)(y+5) r, \\
B_{4}= & \frac{1}{7 \cdot 3^{5} 2^{13}}(y-1)\left(145 y^{3}-4575 y^{2}+58143 y-211825\right)+ \\
& +\frac{5}{3^{5} 2^{8}}(y-19)(y+2)(y+5)(y-1) r, \quad \ldots,
\end{aligned}
$$

где

$$
r=\frac{\delta_{1}-\delta_{2}}{3 \sigma-7 \delta}
$$

Можно видеть, что при таком выборе внешних размерностей коэффициент $B_{2}$ является однозначным, в то время как неоднозначность в старших $B_{k}$ исчезает, если 
выбрать $y=1,-2,-5$, поскольку в этих случаях обращается в ноль коэффициент перед $r$. Заметим теперь, что внешние размерности равны

$$
\begin{array}{lll}
\left(\Delta_{3}, \Delta_{4}\right)=(0,1 / 2) & \text { при } & y=1, \\
\left(\Delta_{3}, \Delta_{4}\right)=(1 / 16,1 / 16) & \text { при } & y=-2, \\
\left(\Delta_{3}, \Delta_{4}\right)=(1 / 2,0) & \text { при } & y=-5
\end{array}
$$

и в точности совпадают с размерностями модели Изинга - модели с центральным зарядом $c=1 / 2$ и тремя примарными операторами с размерностями $0,1 / 2$ и $1 / 16$.

Таким образом, в модели Изинга мы наблюдаем новое явление: коэффициент перед $r$ обращается в ноль, т. е. зависимость от направления подхода к точке модели Изинга исчезает, несмотря на то что эта точка лежит на локусе неоднозначностей.

На самом деле этот факт остается верным и для других минимальных моделей (см. пример $c=1$ в приложении А), и, таким образом (если нет других примеров этого типа), он может служить еще одним определением минимальных моделей, полностью на уровне пертурбативных конформных блоков.

Посмотрим, как это работает для разных четырехточечных конформных блоков в окрестности точки модели Изинга. Имеем следующее:

если $\Delta_{\mathrm{e}}^{(0)}=1 / 16, \Delta^{(0)}=1 / 2$, то

$$
\begin{aligned}
& B_{2}=\frac{\frac{9}{32}(2 \sigma+7 \delta) \epsilon+O\left(\epsilon^{2}\right)}{2(2 \sigma+7 \delta) \epsilon+O\left(\epsilon^{2}\right)}=\frac{9}{64}+O(\epsilon), \\
& B_{3}=\frac{\frac{75}{128}(2 \sigma+7 \delta)(3 \sigma-7 \delta) \epsilon^{2}+O\left(\epsilon^{3}\right)}{6(2 \sigma+7 \delta)(3 \sigma-7 \delta) \epsilon^{2}+O\left(\epsilon^{3}\right)}=\frac{25}{256}+O(\epsilon), \\
& B_{4}=\frac{\frac{502047}{2048}(2 \sigma+7 \delta)(3 \sigma-7 \delta) \epsilon^{2}+O\left(\epsilon^{3}\right)}{12 \cdot 273 \cdot(2 \sigma+7 \delta)(3 \sigma-7 \delta) \epsilon^{2}+O\left(\epsilon^{3}\right)}=\frac{613}{8192}+O(\epsilon), \ldots ;
\end{aligned}
$$

если $\Delta_{\mathrm{e}}^{(0)}=1 / 16, \Delta^{(0)}=0$, то

$$
\begin{aligned}
& B_{2}=\frac{\frac{1}{32} \delta \epsilon+O\left(\epsilon^{2}\right)}{2 \delta \epsilon+O\left(\epsilon^{2}\right)}=\frac{1}{64}+O(\epsilon) \\
& B_{3}=\frac{\frac{15}{32} \delta \epsilon+O\left(\epsilon^{2}\right)}{30 \delta \epsilon+O\left(\epsilon^{2}\right)}=\frac{1}{64}+O(\epsilon) \\
& B_{4}=\frac{-\frac{257985}{4096} \delta \epsilon+O\left(\epsilon^{2}\right)}{-4410 \delta \epsilon+O\left(\epsilon^{2}\right)}=\frac{117}{8192}+O(\epsilon)
\end{aligned}
$$

и снова отсутствует зависимость от $r$.

За дальнейшими деталями о модели Изинга мы отсылаем к приложению Б.

5.7. Резюме. В настоящем разделе мы начали детальное изучение нулей Каца, где расположено большинство традиционно изучаемых конформных моделей, и где вблизи дивизора возникает стандартная неоднозначность, мешающая определению конформного блока, и пертурбативного, и непертурбативного, как хорошо определенного предела несингулярных выражений. Мы видели в разделе 4, что эта неоднозначность может объяснить явную разницу между известными конформными блоками в точке Ашкина-Теллера. На самом деле мы уверены, что понимание этой 
структуры вблизи дивизора будет важным для дальнейшего развития непертурбативной конформной теории поля. Как мы объясняли, эта структура интересна даже на первый взгляд. А именно, мы описали четыре нетривиальных явления, специфичных для конформных блоков и подчеркивающих, что имеется далеко не общее поведение вблизи сингулярности - скорее наоборот, поведение подогнано очень специальным образом, который хотелось бы лучше понять и интерпретировать.

Итак, если изучать ноль Каца на уровне $l$, то соответствующая сингулярность появляется впервые в коэффициенте $B_{l}$, и, чтобы избавиться от сингулярности, нужно подогнать внешние размерности $\Delta_{i}$ так, чтобы числитель $N B_{l}$ этого коэффициента также стал равным нулю. Тогда полюс присутствует также во всех старших коэффициентах $B_{k}$ c $k \geqslant l$. Но важно отметить следующее

ЯвлЕниЕ 1. В конформных блоках имеется вложенная структура: ноль числителя $N B_{l}$ в полюсе также присутствует во всех старших числителях $N B_{k}$ с $k \geqslant l$. Это означает, что когда ноль Каца простой, этого достаточно для того, чтобы подогнать внешние размерности только один раз, в первом подходящем коэффициенте $B_{l}$, и тогда полный конформный блок несингулярен - ноль первого подходящего числителя в простом нуле Каца наследуется всеми остальными числителями.

Однако во многих интересных случаях значений внутренней (промежуточной) размерности $\Delta$ ноль детерминанта Каца не является простым, разные нули знаменателей коэффициентов $B_{k}$ c $k \geqslant l$ "случайно" совпадают для всех конформных теорий с рациональными центральными зарядами. Тогда возникает следующее

ЯвлЕниЕ 2. Вложенная структура нарушается в том смысле, что ноль в числителе конформного блока остается простым. Таким образом, для случайно совпадающих нулей Каца мы обнаруживаем "голые сингулярности", подобные сингулярностям в функциях общего положения на пространстве модулей.

Однако имеется заметное исключение из этой пессимистической картины, а именно

ЯвлЕниЕ 3. Вложенная структура обычно восстанавливается, когда мы добиваемся дополнительного сокращения кратного полюса в первом подходящем коэффициенте $B_{l}$. В этом случае промежуточная размерность $\Delta$ такова, что нули Каца не простые, но один и тот же выбор внешних $\Delta_{i}$ дает во всех числителях нули правильного порядка, позволяющие сократить сингулярность. Тогда неоднозначность конформного блока кусочно-универсальна: если при некотором $l_{1}$ возникает ноль Каца, он становится двойным нулем при некотором $l_{2}$ и т. д. (если возникает "случайный" двойной полюс, рано или поздно возникнут все старшие кратности), все $B_{k}$ с $k<l_{1}$ однозначны, $B_{k}$ с $l_{1} \leqslant k<l_{2}$ являются универсальными линейными функциями одного параметра, описывающего приближение к локусу сингулярностей, коэффициенты $B_{k}$ с $l_{2} \leqslant k<l_{3}$ являются универсальными функциями, которые представляют собой линейные комбинации двух параметров и т. д.

Более того имеет место

Явление 4. В большинстве точек минимальных моделей неоднозначность вблизи дивизора исчезает: предел не зависит от направления в пространстве модулей, по которому мы подходим к точкам минимальных моделей. Это, вероятно, наиболее впечатляющая демонстрация того, насколько минимальные модели действительно специфичны с точки зрения свойств конформного блока, но связь этих свойств со 
многими другими (подобными конечности конформного блока, что подразумевается конформным бутстрапом в этих точках) все еще ждет своего понимания.

Неясно, описывают ли эти четыре явления исчерпывающим образом особенности поведения конформного блока на дивизоре Каца даже в простейшем случае сферической 4-точки. Очень интересно было бы обобщить это исследование на большее число точек и старшие рода. И, конечно, ключевой вопрос - включение непертурбативных поправок. Все это необходимо аккуратно изучить.

\section{6. НУЛЕВЫЕ ВЕКТОРЫ, УРАВНЕНИЯ И СКРЫТЫЕ ПАРАМЕТРЫ}

В предыдущих разделах мы изучили неоднозначности, которые появляются на локусе сингулярностей конформного блока и прояснили их источник и особые свойства. В настоящем разделе мы рассмотрим специальный конформный блок, когда некоторые из внешних размерностей отвечают вырожденному вектору, поскольку в этом случае можно анализировать конформный блок не как ряд, но как пространство решений дифференциального уравнения. Следовательно, можно проверить, есть ли возможность найти некоторые непертурбативные скрытые параметры, т. е. может ли конформный блок быть представлен линейной комбинацией решений с произвольными коэффициентами.

Мы начинаем с простейшего примера вектора, вырожденного на втором уровне. Этот вектор имеет вид $\widetilde{V}=\left(\xi L_{-1}^{2}-L_{-2}\right) V_{\Delta}$, и существуют два нетривиальных условия: $L_{1} \widetilde{V}=0$ и $L_{2} \widetilde{V}=0$. Они подразумевают соответственно, что

$$
\xi=\frac{3}{2(2 \Delta+1)} \quad \text { и } \quad 8 \Delta+c=12 \xi \Delta
$$

или вместе

$$
\Delta=\frac{5-c \pm \sqrt{(c-1)(c-25)}}{16} .
$$

Параметризуя центральный заряд и размерности, как в (3.23), мы получаем четыре решения:

$$
\begin{array}{llll}
\alpha=\frac{1}{2 b}, & \alpha=-\frac{b}{2}, & \alpha=\frac{3 b}{2}-\frac{1}{b}, & \alpha=b-\frac{3}{2 b}, \\
\xi=b^{2}, & \xi=\frac{1}{b^{2}}, & \xi=b^{2}, & \xi=\frac{1}{b^{2}} .
\end{array}
$$

Далее мы работаем с первым из этих четырех решений (в рамке), так что изначальное примарное поле (вектор старшего веса вырожденного модуля Верма) имеет размерность

$$
\Delta_{1 / 2 b}=-\frac{1}{2}+\frac{3}{4 b^{2}}
$$

Конформные тождества Уорда подразумевают, что четырехточечные корреляторы $\Psi_{4}(x, \bar{x})$ со вставкой этого вырожденного примарного поля в точке $x$ удовлетворяют специальному дифференциальному уравнению (см. работы [1])

$$
\left\{b^{2} x(x-1) \partial_{x}^{2}+(2 x-1) \partial_{x}+\Delta_{1 / 2 b}+\frac{\Delta_{1}}{x}-\frac{\Delta_{3}}{x-1}-\Delta_{4}\right\} \Psi_{4}(x)=0,
$$


где мы опустили зависимость от $\bar{x}$. В свободно-полевой формулировке конформной теории поля данное условие налагается автоматически [61], это также легко увидеть из представления Доценко-Фатеева соответствующего конформного блока в виде $\beta$-ансамбля [61].

Сопрягая при помощи фактора $x^{\alpha}(1-x)^{\beta}$ со специально подобранными $\alpha$ и $\beta$, можно превратить (6.5) в обыкновенное гипергеометрическое уравнение с решением

$$
\begin{gathered}
\Psi_{4}(x)=x^{\alpha_{1} / b}(1-x)^{\alpha_{3} / b} F(A, B ; C ; x), \\
A=\frac{1}{2 b^{2}}+\frac{\alpha_{1}}{b}+\frac{\alpha_{2}}{b}-\frac{\alpha_{3}}{b}, \quad B=\frac{1}{b} \sum_{i=1}^{3} \alpha_{i}+2 \Delta_{1 / 2 b}, \quad C=\frac{1}{b^{2}}+\frac{2 \alpha_{1}}{b},
\end{gathered}
$$

где $F(A, B ; C ; x)$ - гипергеометрическая функция.

Формулы (6.5), (6.6) согласованы с общими формулами (3.19), (3.20), только если размерности $\Delta_{1}$ и $\Delta$ связаны (в параметризации (3.23)) следующим правилом слияния:

$$
\alpha=\alpha_{1} \pm \frac{1}{2 b}
$$

где знаки плюс и минус отвечают двум линейно независимым решениям уравнения (6.5), а в случае знака минус в (6.7), нужно выбрать в $(6.6)$ вместо $F(A, B ; C ; x)$ другое решение гипергеометрического уравнения, так что $\alpha_{1} \rightarrow b-1 / b-\alpha_{1}$ в функции $\Psi_{4}(x)(6.6)$.

Нетрудно проверить непосредственно, что конформный блок

$$
\begin{aligned}
\mathcal{B}_{\Delta_{\alpha}}^{(1,1 / 2 b ; 34)}(x) & =x^{\Delta_{\alpha}-\Delta_{1}-\Delta_{1 / 2 b}}\left(1+\frac{\left(\Delta_{\alpha}+\Delta_{1 / 2 b}-\Delta_{1}\right)\left(\Delta_{\alpha}+\Delta_{3}-\Delta_{4}\right)}{2 \Delta_{\alpha}} x+\cdots\right) \stackrel{(6.7)}{=} \\
& \stackrel{(6.7)}{=} \Psi_{4}(x),
\end{aligned}
$$

и это дает решение уравнения (6.5). Формула (3.1) теперь принимает вид

$$
\begin{gathered}
\left\langle V_{1}(0) V_{1 / 2 b}(x) V_{3}(1) V_{4}(\infty)\right\rangle=\sum_{\Delta, \bar{\Delta}} C_{1,1 / 2 b}^{\Delta, \bar{\Delta}} C_{34}^{\Delta, \bar{\Delta}} \mathcal{B}_{\Delta}^{(1,1 / 2 b ; 34)}(x) \overline{\mathcal{B}}_{\bar{\Delta}}^{(1,1 / 2 b ; 34)}(\bar{x})= \\
=\sum_{\substack{\alpha=\alpha_{1} \pm 1 / 2 b \\
\bar{\alpha}=\bar{\alpha}_{1} \pm 1 / 2 b}} C_{1,1 / 2 b}^{\Delta_{\alpha}, \Delta_{\bar{\alpha}}} C_{34}^{\Delta_{\alpha}, \Delta_{\bar{\alpha}}} \mathcal{B}_{\Delta}^{(1,1 / 2 b ; 34)}(x) \overline{\mathcal{B}}_{\bar{\Delta}}^{(1,1 / 2 b ; 34)}(\bar{x}),
\end{gathered}
$$

так как только при выполнении соотношения (6.7) структурная константа $C_{1,1 / 2 b}^{\Delta_{\alpha}}$ не равна нулю [1]. Здесь мы получили этот факт косвенным образом, решая уравнение для коррелятора. Этот факт можно вывести и непосредственно, используя представление конформного блока в виде $\beta$-ансамбля [61].

Таким образом, два решения уравнения для вырожденного конформного блока описывают два разных конформных блока, два единственных блока с ненулевыми структурными константами. Можно также легко увидеть, как получается условие (6.7) из их асимптотики: поскольку конформный блок ведет себя при малых $x$, как $x^{\Delta-\Delta_{1}-\Delta_{2}}$, равенство (6.7) получается из асимптотического поведения $\Psi_{4}^{(1)}(x) \sim x^{\alpha_{1} / b}$ и $\Psi_{4}^{(2)}(x) \sim x^{1-1 / b^{2}-\alpha_{1} / b}$. Эта система двух конформных блоков 
самосогласованна - она выдерживает модулярное преобразование (3.3) $x \rightarrow 1-x$, $\Delta_{1} \leftrightarrow \Delta_{3}$. Действительно (см. справочник [60], формулы $9.131(1,2)$ ),

$$
\begin{aligned}
& \Psi_{4}^{(1)}\left(\Delta_{3}, \Delta_{2}, \Delta_{1}, \Delta_{4} ; 1-x\right)= \\
& =\frac{\Gamma\left(\frac{2 \alpha_{3}}{b}+\frac{1}{b^{2}}\right) \Gamma\left(\frac{1}{b^{2}}+\frac{2 \alpha_{1}}{b}-1\right)}{\Gamma\left(\frac{3}{2 b^{2}}-1+\frac{\alpha_{1}}{b}+\frac{\alpha_{3}}{b}+\frac{\alpha_{4}}{b}\right) \Gamma\left(\frac{1}{2 b^{2}}+\frac{\alpha_{1}}{b}+\frac{\alpha_{3}}{b}-\frac{\alpha_{4}}{b}\right)} \Psi_{4}^{(1)}\left(\Delta_{1}, \Delta_{2}, \Delta_{3}, \Delta_{4} ; x\right)+ \\
& \quad+\frac{\Gamma\left(\frac{2 \alpha_{3}}{b}+\frac{1}{b^{2}}\right) \Gamma\left(1-\frac{1}{b^{2}}-\frac{2 \alpha_{1}}{b}\right)}{\Gamma\left(1-\frac{1}{2 b^{2}}+\frac{\alpha_{3}}{b}-\frac{\alpha_{1}}{b}-\frac{\alpha_{4}}{b}\right) \Gamma\left(\frac{1}{2 b^{2}}+\frac{\alpha_{3}}{b}+\frac{\alpha_{4}}{b}-\frac{\alpha_{1}}{b}\right)} \Psi_{4}^{(2)}\left(\Delta_{1}, \Delta_{2}, \Delta_{3}, \Delta_{4} ; x\right), \\
& \Psi_{4}^{(2)}\left(\Delta_{3}, \Delta_{2}, \Delta_{1}, \Delta_{4} ; 1-x\right)= \\
& =\frac{\Gamma\left(2-\frac{2 \alpha_{3}}{b}-\frac{1}{b^{2}}\right) \Gamma\left(\frac{1}{b^{2}}-1+\frac{2 \alpha_{1}}{b}\right)}{\Gamma\left(\frac{1}{2 b^{2}}+\frac{\alpha_{1}}{b}+\frac{\alpha_{4}}{b}-\frac{\alpha_{3}}{b}\right) \Gamma\left(1-\frac{1}{2 b^{2}}+\frac{\alpha_{1}}{b}-\frac{\alpha_{3}}{b}-\frac{\alpha_{4}}{b}\right)} \Psi_{4}^{(1)}\left(\Delta_{1}, \Delta_{2}, \Delta_{3}, \Delta_{4} ; x\right)+ \\
& \quad+\frac{\Gamma\left(2-\frac{2 \alpha_{3}}{b}-\frac{1}{b^{2}}\right) \Gamma\left(1-\frac{1}{b^{2}}-\frac{2 \alpha_{1}}{b}\right)}{\Gamma\left(2-\frac{3}{2 b^{2}}-\frac{\alpha_{1}}{b}-\frac{\alpha_{3}}{b}-\frac{\alpha_{4}}{b}\right) \Gamma\left(1-\frac{1}{2 b^{2}}+\frac{\alpha_{1}}{b}+\frac{\alpha_{3}}{b}-\frac{\alpha_{4}}{b}\right)} \Psi_{4}^{(2)}\left(\Delta_{1}, \Delta_{2}, \Delta_{3}, \Delta_{4} ; x\right),
\end{aligned}
$$

т. е. модулярное ядро в этом случае есть следующая матрица размера $2 \times 2$ :

$$
\left(\begin{array}{cc}
\frac{\Gamma\left(\frac{2 \alpha_{3}}{b}+\frac{1}{b^{2}}\right) \Gamma\left(\frac{1}{b^{2}}+\frac{2 \alpha_{1}}{b}-1\right)}{\Gamma\left(\frac{3}{2 b^{2}}-1+\frac{\alpha_{1}}{b}+\frac{\alpha_{3}}{b}+\frac{\alpha_{4}}{b}\right) \Gamma\left(\frac{1}{2 b^{2}}+\frac{\alpha_{1}}{b}+\frac{\alpha_{3}}{b}-\frac{\alpha_{4}}{b}\right)} & \frac{\Gamma\left(\frac{2 \alpha_{3}}{b}+\frac{1}{b^{2}}\right) \Gamma\left(1-\frac{1}{b^{2}}-\frac{2 \alpha_{1}}{b}\right)}{\Gamma\left(1-\frac{1}{2 b^{2}}+\frac{\alpha_{3}}{b}-\frac{\alpha_{1}}{b}-\frac{\alpha_{4}}{b}\right) \Gamma\left(\frac{1}{2 b^{2}}+\frac{\alpha_{3}}{b}+\frac{\alpha_{4}}{b}-\frac{\alpha_{1}}{b}\right)} \\
\frac{\Gamma\left(2-\frac{2 \alpha_{3}}{b}-\frac{1}{b^{2}}\right) \Gamma\left(\frac{1}{b^{2}}-1+\frac{2 \alpha_{1}}{b}\right)}{\Gamma\left(\frac{1}{2 b^{2}}+\frac{\alpha_{1}}{b}+\frac{\alpha_{4}}{b}-\frac{\alpha_{3}}{b}\right) \Gamma\left(1-\frac{1}{2 b^{2}}+\frac{\alpha_{1}}{b}-\frac{\alpha_{3}}{b}-\frac{\alpha_{4}}{b}\right)} & \frac{\Gamma\left(2-\frac{2 \alpha_{3}}{b}-\frac{1}{b^{2}}\right) \Gamma\left(1-\frac{1}{b^{2}}-\frac{2 \alpha_{1}}{b}\right)}{\Gamma\left(2-\frac{3}{2 b^{2}}-\frac{\alpha_{1}}{b}-\frac{\alpha_{3}}{b}-\frac{\alpha_{4}}{b}\right) \Gamma\left(1-\frac{1}{2 b^{2}}+\frac{\alpha_{1}}{b}+\frac{\alpha_{3}}{b}-\frac{\alpha_{4}}{b}\right)}
\end{array}\right) .
$$

Мы демонстрируем, как все это работает в минимальных моделях, на примере $c=1$ и $c=1 / 2$ (модель Изинга) в приложениях А и Б соответственно.

Подобным образом можно работать с конформными блоками, вырожденными на старших уровнях. В этом случае порядок соответствующего дифференциального уравнения выше, но снова в точности равен числу конформных блоков с ненулевыми структурными константами. Модулярная матрица также соответственно увеличивает свой размер (см. примеры в работах [1]). Следовательно, никаких скрытых параметров таким образом не возникает. На самом деле это не очень удивительно, поскольку дифференциальные уравнения - это уравнения по переменной $x, \mathrm{a}$, как мы уже подчеркивали, не следует ожидать, что поведение конформного блока может зависеть от непертурбативных (скрытых) параметров.

\section{7. ЗАКЛЮЧЕНИЕ}

Основная цель настоящей работы - подтолкнуть читателя к изучению непертурбативного конформного блока как функции всех его переменных: координат, внешних и внутренней размерностей и центрального заряда. Первый вопрос здесь состоит в том, имеются ли дополнительные параметры, кроме уже перечисленных, от которых непертурбативная величина обычно зависит и которые не видны на пертурбативном уровне, подобно тета-углу в инстантонных вычислениях. В истории с конформным блоком основная неоднозначность содержится в общей нормировке, которая может быть функцией от размерностей и центрального заряда (и, таким образом, может содержать бесконечно много дополнительных параметров). Отсутствие контроля за такими нормировочными множителями является основной текущей проблемой при соотнесении разных эффективных непертурбативных подходов, 
скажем, к построению модулярного ядра: $S L_{q}(2)$-метода Понсо-Тешнера [41], матрично-модельного подхода [57], раскрывающего явление Стокса (пересечения стенок) и связывающего кластерные переменные с чек-экспонентами [62], и метода уравнения Пенлеве [58].

В настоящей работе мы не решали проблему зависимости от $\Delta$ напрямую - вместо этого мы попытались искать непертурбативные параметры, рассматривая зависимость от $x$ простейшего (четырехточечного сферического) конформного блока. В основном мы рассмотрели два очевидных сюжета: во-первых, несогласованность известных явных ответов в точке Ашкина-Теллера - непертурбативного ответа Замолодчикова и эллиптического интеграла (они являются разными функциями от $x$ ) - и, во-вторых, дифференциальные выражения старшего порядка по $x$, которым удовлетворяет конформный блок, когда один из операторов вырожден (например, в модели Изинга и других минимальных моделях). В обоих случаях, как кажется, подразумевается существование дополнительных параметров. Как мы объяснили, это, однако, не так. Несогласованность в точке Ашкина-Теллера (и во многих других случаях), в действительности представляет собой просто обычную неоднозначность на дивизоре сингулярностей функции многих переменных (размерностей и центрального заряда), и дополнительные параметры не возникают.

Случай со многими, а не одним решением дифференциального уравнения старшего порядка разрешается не введением дополнительных переменных, а тем фактом, что $B(x)$ в действительности не удовлетворяет таким уравнениям, когда одна внешняя размерность фиксирована. На самом деле это уравнение верно для конформного блока, только когда внутренняя размерность $\Delta$ также фиксирована [61] (это очень важная черта конформного блока, которая часто пропускается или недооценивается). В результате дополнительные решения на самом деле описывают не неоднозначность функции $B_{\Delta}(x)$, но другие конформные блоки $B_{\Delta^{\prime}}(x)$ с другими допустимыми значениями внутренней размерности $\Delta^{\prime}$ : их имеется ровно столько, каков уровень, на котором вырожден вершинный оператор, и какой порядок у дифференциального уравнения.

Итак, мы не нашли указаний на дополнительные непертурбативные параметры в зависимости конформного блока от $x$. Это могло показаться очевидным с самого начала: как мы упоминали, имеется вера в то, что четырехточечный сферический конформный блок является в действительности функцией Белого (т. е. обладает только несущественными особенностями в трех точках $x=0,1, \infty$, и порядки ветвления равны целым числам для рациональных конформных моделей). Это, в свою очередь, можно ассоциировать либо с тем фактом, что двумерная конформная теория поля есть по существу теория свободных полей [1], так что в реальности нет никакого взаимодействия и нет никакой причины для существования реальных непертурбативных эффектов, либо с тем фактом, что коррелятор конформный и, следовательно, никакая нетривиальная зависимость от единственного размерного параметра $x$ недопустима.

Мы верим, что наше простое рассмотрение проливает некоторый свет на проблему непертурбативных конформных блоков и может помочь привлечь новое внимание к этой крайне интересной проблеме. Конформные блоки - критически важные специальные функции струнной эры, и их нужно тщательно изучать и в конечном итоге понять. 


\section{Случай $c=1, \Delta_{\mathrm{e}}=1 / 4$}

\section{ПРИЛОЖЕНИЕ А}

Рассмотрим минимальную модель с $c=1$, которую можно получить из серии минимальных моделей $(m, m+1)$ в пределе $m \rightarrow \infty$ [46]. Нетрудно сконструировать конформный блок из четырех полей с $\Delta_{1,2}=1 / 4$, поскольку они вырожденны на втором уровне. Решая уравнение (6.5) с $b=1$, мы получим два решения, которые соответствуют конформным блокам с размерностями $\Delta=0$,

$$
\begin{aligned}
\left.\mathcal{B}\left(\Delta_{i}, c \mid x\right)\right|_{\Delta_{i}=1 / 4, c=1} & =\frac{1}{\sqrt{x}}\left(1+\frac{x^{2}}{8}+\frac{x^{3}}{8}+\frac{15 x^{4}}{128}+\cdots\right)= \\
& =\frac{1}{2 \sqrt{x}}\left(\sqrt{1-x}+\frac{1}{\sqrt{1-x}}\right),
\end{aligned}
$$

и $\Delta=1$,

$$
\left.\mathcal{B}\left(\Delta_{i}, c \mid x\right)\right|_{\Delta_{i}=1 / 4, c=1}=\sqrt{x}\left(1+\frac{x}{2}+\frac{3 x^{2}}{8}+\frac{5 x^{3}}{16}+\frac{35 x^{4}}{128}+\cdots\right)=\frac{\sqrt{x}}{\sqrt{1-x}} .
$$

Модулярное преобразование $x \rightarrow 1-x$ действует на дублет

$$
\left(\begin{array}{c}
\frac{1}{2} \sqrt{\frac{1-x}{x}}+\frac{1}{2} \sqrt{\frac{1}{x(1-x)}} \\
\sqrt{\frac{x}{1-x}}
\end{array}\right),
$$

как матрица

$$
\left(\begin{array}{cc}
1 / 2 & 3 / 4 \\
1 & -1 / 2
\end{array}\right)
$$

Из билинейной комбинации этих блоков можно сконструировать модулярный инвариант, подбирая коэффициент:

$$
\frac{1}{|x|}\left|\mathcal{B}_{0}\right|^{2}+\frac{3|x|}{4}\left|\mathcal{B}_{1}\right|^{2}=\frac{|1-x / 2|^{2}+3|x|^{2} / 4}{|x||1-x|} \sim \frac{|x|}{|1-x|}+\frac{|1-x|}{|x|}+\frac{1}{|x(1-x)|} .
$$

Этот ответ совпадает с приведенным в работе [46].

\section{Модель Изинга}

ПРИЛОЖЕНИЕ Б

Критическое поведение двумерной модели Изинга описывается конформной теорией с центральным зарядом $c=1 / 2$, и мы выбираем $b=\sqrt{3} / 2$. Имеются три примарных поля с размерностями

$$
\begin{array}{ll}
\Delta_{I}=0 & \text { (т. е. } \left.\alpha_{I}=0 \text { или } \alpha_{I}=-\sqrt{3} / 6\right), \\
\Delta_{\psi}=1 / 2 & \text { (т. е. } \left.\alpha_{\psi}=\sqrt{3} / 3 \text { или } \alpha_{\psi}=-\sqrt{3} / 2\right), \\
\Delta_{\sigma}=1 / 16 & \text { (т. е. } \left.\alpha_{\sigma}=\sqrt{3} / 12 \text { или } \alpha_{\sigma}=-\sqrt{3} / 4\right) .
\end{array}
$$

Первое поле вырожденно на первом уровне, второе и третье - на втором уровне. В этом случае можно вычислить все конформные блоки. Рассмотрим, к примеpy, $\mathcal{B}\left(\Delta_{1}, \Delta_{2}, \Delta_{3}, \Delta_{4} ; \Delta ; c ; x\right)$ с промежуточной размерностью $\Delta=\Delta_{\psi}=1 / 2$. Это 
дает полюсы на всех уровнях старше первого. Условие сокращения полюса путем подгонки размерностей $\Delta_{1}$ и $\Delta_{2}$ требует, чтобы либо $\Delta_{1}=1 / 2$ и $\Delta_{2}=0$, либо $\Delta_{1}=\Delta_{2}=1 / 16$ (если ограничиться спектром трех вышеприведенных полей). Следовательно, корреляторы полей $\langle\psi \psi I\rangle$ и $\langle\psi \sigma \sigma\rangle$ не равны нулю. Рассматривая подобным образом конформный блок с промежуточной размерностью $\Delta=\Delta_{\sigma}=1 / 16$, можно найти ненулевой коррелятор $\langle\psi \psi I\rangle$. Это фиксирует ОР полей.

Рассмотрим теперь конформный блок с полем $\psi(x)$ в точке $x$ (напомним, что в наших обозначениях это отвечает $\Delta_{2}$, в то время как $\Delta_{1}, \Delta_{3}$ и $\Delta_{4}$ соответствуют полям в точках 0,1 и $\infty$ соответственно). Это поле вырожденно на втором уровне, а конформный блок удовлетворяет дифференциальному уравнению второго порядка при условии $\alpha=\alpha_{1} \pm 1 / 2 b$ (см. раздел 6 ). Данное условие можно удовлетворить только для пар $\left(\alpha, \alpha_{1}\right)$, или $\left(\alpha_{\sigma}, \alpha_{\sigma}\right)$, или $\left(\alpha_{\psi}, \alpha_{I}\right)$, что снова фиксирует ОР.

Рассмотрим коррелятор $\langle\sigma(0) \psi(x) \sigma(1) \psi(\infty)\rangle$. Он описывается значениями $\alpha_{1}=$ $-\sqrt{3} / 4, \alpha_{2}=\sqrt{3} / 3, \alpha_{3}=-\sqrt{3} / 4, \alpha_{4}=-\sqrt{3} / 2$. Соответствующий конформный блок $\mathcal{B}(x)$ удовлетворяет дифференциальному уравнению (6.5):

$$
\left[\frac{3}{4} x(1-x) \partial_{x}^{2}+(2 x-1) \partial_{x}+\frac{1}{16}\left(\frac{1}{x}+\frac{1}{1-x}\right)\right] \mathcal{B}(x)=0 .
$$

Это гипергеометрическое уравнение, оно имеет два решения

$$
\mathcal{B}^{(1)}(x)=\frac{1-2 x}{\sqrt{x(1-x)}}, \quad \mathcal{B}^{(2)}(x)=[x(1-x)]^{1 / 6} F(1 / 3,2 ; 5 / 3 ; x) .
$$

Первое решение соответствует при малых $x$ следующему поведению конформного блока:

$$
c \mathcal{B}^{(1)}(x)=\frac{1}{\sqrt{x}}\left(1-\frac{3}{2} x-\frac{5}{8} x^{2}-\frac{7}{16} x^{3}-\frac{45}{128} x^{4}+\cdots\right) .
$$

Множитель $1 / \sqrt{x}$ приходит из обычного префактора $x^{\Delta-\Delta_{1}-\Delta_{2}}$ конформного блока и подразумевает (как мы обосновали на основе ОР), что $\Delta=\Delta_{\sigma}=1 / 16$. Второе решение должно бы описывать промежуточное поле с размерностью $\Delta=35 / 48$. Данное поле отсутствует в спектре, и это означает, что соответствующая структурная константа равна нулю.

Разложение конформного блока (Б.3) нужно сравнивать с его общим разложением (3.18). Можно проверить, что они совпадают вне зависимости от способа, каким разрешается особенность, т. е., если рассмотреть окрестность точки $\Delta_{1}=1 / 16$, $\Delta_{2}=1 / 2, \Delta_{3}=16, \Delta_{4}=1 / 2, \Delta=1 / 16$, лидирующий порядок по $\epsilon$ совершенно не зависит от пути подхода к сингулярности (см. п. 5.6):

$$
B(x)=1+\sum_{k=1}^{\infty} B_{k} x^{k}=1-\frac{3}{2} x-\frac{5}{8} x^{2}-\frac{7}{16} x^{3}-\frac{45}{128} x^{4}+\cdots .
$$

Заметим, что $\mathcal{B}^{(1)}(x)$ согласованным образом инвариантен относительно преобразования дуальности $x \rightarrow 1-x$. Подобным образом инвариантом является дифференциальное уравнение (Б.1), хотя второе решение не является инвариантом - оно преобразуется через себя и первое решение (см. справочник [60], формула 9.131(2)):

$$
F(1 / 3,2 ; 5 / 3 ; x)=F(1 / 3,2 ; 5 / 3 ; 1-x)+\frac{\Gamma(2 / 3)^{3}}{\sqrt{3} \pi} \frac{1}{\left[x(1-x]^{1 / 6}\right.} \mathcal{B}^{(1)}(x) .
$$

Это означает, что матрица дуальности треугольная. 
Рассмотрим теперь другой возможный четырехточечный коррелятор в этой теории: $\langle\sigma \sigma \sigma \sigma\rangle$. Можно подобным образом выписать дифференциальное уравнение

$$
\left[\frac{4}{3} x(1-x) \partial_{x}^{2}+(2 x-1) \partial_{x}+\frac{1}{16}\left(\frac{1}{x}+\frac{1}{1-x}\right)\right] \mathcal{B}(x)=0 .
$$

$\mathrm{У}$ этого уравнения имеются два решения

$$
\mathcal{B}^{(1)}(x)=\frac{\sqrt{\sqrt{x}+1}+\sqrt{\sqrt{x}-1}}{\left[x(1-x]^{1 / 8}\right.}, \quad \mathcal{B}^{(2)}(x)=\frac{\sqrt{\sqrt{x}+1}-\omega \sqrt{\sqrt{x}-1}}{\left[x(1-x]^{1 / 8}\right.},
$$

где $\omega \equiv e^{ \pm \pi i / 2}-$ квадратный корень из -1 (знак плюс или минус зависит от выбранной ветви $\sqrt{x}$ ). Первое решение имеет разложение при малых $x$ вида

$$
\mathcal{B}^{(1)}(x) \sim \frac{1}{x^{1 / 8}}\left(1+\frac{1}{64} x^{2}+\frac{1}{64} x^{3}+\frac{117}{8192} x^{4}+\frac{53}{4096} x^{5}+\cdots\right)
$$

и соответствует промежуточному полю $I$ с размерностью $\Delta=\Delta_{I}=0$. Второе решение имеет разложение при малых $x$, которое записывается как

$$
\mathcal{B}^{(2)}(x) \sim \frac{\sqrt{x}}{x^{1 / 8}}\left(1+\frac{1}{4} x+\frac{9}{64} x^{2}+\frac{25}{256} x^{3}+\frac{613}{8192} x^{4}\right) .
$$

Эти два разложения, как и ранее, воспроизводят правильный результат (Б.3) независимо от способа разрешения особенности.

Поскольку в этом случае оба решения отвечают “физическим” конформным блокам, не удивительно, что преобразование дуальности действует на эти два решения, как умножение на матрицу:

$$
\begin{aligned}
& \mathcal{B}^{(1)}(1-x)=\frac{1}{2}\left(\mathcal{B}^{(1)}(x)+\frac{\omega}{2} \mathcal{B}^{(2)}(x)\right), \\
& \mathcal{B}^{(2)}(1-x)=\sqrt{2}\left(\mathcal{B}^{(1)}(x)-i \frac{\omega}{2} \mathcal{B}^{(2)}(x)\right),
\end{aligned}
$$

т. е. матрица дуальности равна

$$
\sqrt{2}\left(\begin{array}{cc}
1 & \omega / 2 \\
2 & -i \omega
\end{array}\right)
$$

Последний нетривиальный коррелятор в модели Изинга - это $\langle\psi \psi \psi \psi\rangle$. Он описывается дифференциальным уравнением

$$
\left[\frac{3}{4} x(1-x) \partial_{x}^{2}+(2 x-1) \partial_{x}+\frac{1}{2}\left(\frac{1}{x}+\frac{1}{1-x}\right)\right] \mathcal{B}(x)=0 .
$$

Снова только одно из двух решений этого уравнения отвечает модели Изинга, а именно

$$
\mathcal{B}(x)=\frac{1-x+x^{2}}{x(1-x)}
$$

Это решение является модулярным инвариантом, и его разложение при малых $x$ дает конформный блок (Б.3) вне зависимости от способа разрешения особенности:

$$
\mathcal{B}(x) \sim \frac{1}{x}\left(1+x^{2}+x^{3}+x^{4}+\cdots\right),
$$

где общий множитель $1 / x$ подразумевает, что этот конформный блок описывает промежуточное поле размерности $\Delta=\Delta_{I}=0$, как это и должно быть. 
Благодарности. Наша работа частично поддержана Программой поддержки ведущих научных школ (грант НШ-1500.2014.2 (А. Мир. и А. Мор.)) и РФФИ (гранты № 13-02-00457 (А. Мир.), 13-02-00478 (А. Мор.), совместные гранты № 13-02-91371-ST и 14-01-92691-Ind (А. Мир. и А. Мор.)), а также Brazilian Council for Scientific and Technological Development (A. Мор.). Исследование Х. И. частично поддержано Министерством образования, науки и культуры Японии (грант Grant-in-Ad for Scientific Research (23540316)). Мы благодарны за поддержку программе двустороннего сотрудничества JSPS RFBR "Synthesis of integrabilities arising from gauge-string duality" (грант FY2010-2011: 12-02-92108-Яф_a).

\section{Список литературы}

[1] A. A. Belavin, A. M. Polyakov, A. B. Zamolodchikov, Nucl. Phys. B, 241:2 (1984), 333-380; А.Б. Замолодчиков, Ал. Б. Замолодчиков, Конформная теория поля и критические явления в двумерных системах, МНЦМО, 2009; L. Alvarez-Gaumé, Helv. Phys. Acta, 64:4 (1991), 359-626; P. Di Francesco, P. Mathieu, D. Sénéchal, Conformal Field Theory, Springer, New York, 1997.

[2] M. Sato, RIMS. Kokyuroku, 439 (1981), 30-46; E. Date, M. Jimbo, M. Kashiwara, T. Miwa, "Transformation groups for soliton equations", Nonlinear Integrable Systems - Classical Theory and Quantum Theory (Kyoto, 1981), eds. M. Jimbo, T. Miwa, World Sci., Singapore, 1983, 39-119; M. Jimbo, T. Miwa, Publ. Res. Inst. Math. Sci., Kyoto Univ., 19:3 (1983), 943-1001.

[3] J. Wess, B. Zumino, Phys. Lett. B, 37:1 (1971), 95-97; E. Witten, Nucl. Phys. B, 223:2 (1983), 422-421; Commun. Math. Phys., 92:4 (1984), 455-472; С. П. Новиков, Докл. АН CCCP, 260 (1981), 31-35; УMH, 37:5(227) (1982), 3-49.

[4] A. Gerasimov, A. Morozov, M. Olshanetsky, A. Marshakov, S. Shatashvili, Internat. J. Modern Phys. A, 5:13 (1990), 2495-2589.

[5] A. Gerasimov, S. Khoroshkin, D. Lebedev, A. Mironov, A. Morozov, Internat. J. Modern Phys. A, 10:18 (1995), 2589-2614, arXiv: hep-th/9405011; S. M. Kharchev, S. M. Khoroshkin, D. R. Lebedev, TMФ, 104:1 (1995), 144-157, arXiv: q-alg/9501013; А. Д. Миронов, ТМФ, 114:2 (1998), 163-232; A. Mironov, "Quantum deformations of $\tau$-functions, bilinear identities, and representation theory", Symmetries and Integrability of Difference Equations (Estérel, PQ, May 22-29, 1994), CRM Proceedings and Lecture Notes, 9, eds. D. Levi, L. Vinet, P. Winternitz, AMS, Providence, RI, 1996, 219-237, arXiv: hep-th/9409190.

[6] S.-S. Chern, J. Simons, Ann. Math. (2), 99:1 (1974), 48-69.

[7] E. Witten, Commun. Math. Phys., 121:3 (1989), 351-399.

[8] E. Guadagnini, M. Martellini, M. Mintchev, "Chern-Simons field theory and quantum groups", Quantum Groups, Proceedings of the 8th International Workshop on Mathematical Physics (Arnold Sommerfeld Institute, Clausthal, Germany, 19-26 July, 1989), Lecture Notes in Physics, 370, eds. H.-D. Doebner, J.-D. Hennig, Springer, Berlin, 307-317; Phys. Lett. B, 235:3-4 (1990), 275-281; N. Yu. Reshetikhin, V. G. Turaev, Commun. Math. Phys., 127:1 (1990), 1-26.

[9] P. Ramadevi, T. R. Govindarajan, R. K. Kaul, Nucl. Phys. B, 402:1-2 (1993), 548-566, arXiv: hep-th/9212110; 422:1-2 (1994), 291-306, arXiv: hep-th/9312215.

[10] A. Morozov, A. Smirnov, Nucl. Phys. B, 835:3 (2010), 284-313, arXiv: 1001.2003.

[11] A. Mironov, A. Morozov, And. Morozov, "Character expansion for HOMFLY polynomials. I. Integrability and difference equations", Strings, Gauge Fields, and the Geometry Behind: The Legacy of Maximilian Kreuzer, eds. A. Rebhan, L. Katzarkov, J. Knapp, R. Rashkov, E. Scheidegger, World Sci., Singapore, 2013, 101-118, arXiv: 1112.5754; JHEP, 03 (2012), 034, 34 pp., arXiv: 1112.2654. 
[12] M. Atiyah, The Geometry and Physics of Knots, Cambridge Univ. Press, Cambridge, 1990.

[13] L. Alday, D. Gaiotto, Y. Tachikawa, Lett. Math. Phys., 91:2 (2010), 167-197, arXiv: 0906.3219.

[14] N. Wyllard, JHEP, 11 (2009), 002, 22 pp., arXiv: 0907.2189.

[15] A. Mironov, A. Morozov, Nucl. Phys. B, 825:1-2 (2009), 1-37, arXiv: 0908.2569.

[16] R. Dijkgraaf, C. Vafa, Toda Theories, matrix models, topological strings, and $N=2$ gauge systems, arXiv: 0909.2453; T. Eguchi, K. Maruyoshi, Penner type matrix model and Seiberg-Witten theory, arXiv: 0911.4797; JHEP, 2 (2010), 022, 21 pp., arXiv: 1006.0828; H. Itoyama, K. Maruyoshi, T. Oota, Prog. Theor. Phys., 123:6 (2010), 957-987, arXiv: 0911.4244.

[17] A. Mironov, A. Morozov, Sh. Shakirov, JHEP, 02 (2010), 030, 26 pp., arXiv: 0911.5721; Internat. J. Modern Phys. A, 25:16 (2010), 3173-3207, arXiv: 1001.0563; JHEP, 03 (2011), 102, 25 pp., arXiv: 1011.3481; A. Mironov, Al. Morozov, And. Morozov, Nucl. Phys. B, 843:2 (2011), 534-557, arXiv: 1003.5752.

[18] A. Mironov, A. Morozov, Sh. Shakirov, JHEP, 02 (2011), 067, 41 pp., arXiv: 1012.3137.

[19] V. A. Alba, V. A. Fateev, A. V. Litvinov, G. M. Tarnopolsky, Lett. Math. Phys., 98:1 (2011), 33-64, arXiv: 1012.1312; A. Belavin, V. Belavin, Nucl. Phys. B, 850:1 (2011), 199-213, arXiv: 1102.0343; S. Mironov, An. Morozov, Y. Zenkevich, Писъма в ЖЭЭФ, 99:2 (2014), 115-119, arXiv: 1312.5732; Y. Matsuo, C. Rim, H. Zhang, JHEP, 09 (2014), 028, 27 pp., arXiv: 1405.3141.

[20] H. Awata, Y. Yamada, JHEP, 01 (2010), 125, 11 pp., arXiv: 0910.4431; Prog. Theor. Phys., 124:2 (2010), 227-262, arXiv: 1004.5122; S. Yanagida, J. Math. Phys., 51:12 (2010), 123506, 13 pp., arXiv: 1005.0216.

[21] A. Mironov, A. Morozov, Sh. Shakirov, A. Smirnov, Nucl. Phys. B, 855:1 (2012), 128-151, arXiv: 1105.0948.

[22] H. Itoyama, T. Oota, R. Yoshioka, Nucl. Phys. B, 877:2 (2013), 506-537, arXiv: 1308.2068.

[23] A. Losev, N. Nekrasov, S. Shatashvili, Nucl. Phys. B, 534:3 (1998), 549-611, arXiv: hep-th/9711108; "Testing Seiberg-Witten solution", L.D. Faddeev's Seminar on Mathematical Physics, Translations American Mathematical Society. Ser. 2, 201, ed. M. Semenov-Tian-Shansky, AMS, Providence, RI, 2000, 123-135, arXiv: hep-th/9801061; G. Moore, N. Nekrasov, S. Shatashvili, Commun. Math. Phys., 209:1 (2000), 77-95, arXiv: hep-th/9803265; 97-121, arXiv: hep-th/9712241.

[24] N. Nekrasov, Adv. Theor. Math. Phys., 7:5 (2004), 831-864; R. Flume, R. Pogossian, Internat. J. Modern Phys. A, 18:14 (2003), 2541-2563, arXiv: hep-th/0208176; N. Nekrasov, A. Okounkov, "Seiberg-Witten theory and random partitions", The Unity of Mathematics, Progress in Mathematics, 244, eds. P. Etingof, V. Retakh, I. M. Singer, Birkhäuser, Boston, MA, 2006, 525-596, arXiv: hep-th/0306238.

[25] V. Pestun, JHEP, 12 (2012), 067, 40 pp., arXiv: 0906.0638.

[26] A. Mironov, A. Morozov, Phys. Lett. B, 680:2 (2009), 188-194, arXiv: 0908.2190.

[27] M. Bershtein, O. Foda, JHEP, 06 (2014), 177, 26 pp., arXiv: 1404.7075; K. B. Alkalaev, V.A. Belavin, Conformal blocks of $W_{N}$ minimal models and AGT correspondence, arXiv: 1404.7094.

[28] Ал. Б. Замолодчиков, ЖЖЭТФ, 90:5, 1808-1818; ТМФ, 73:1 (1987), 103-110.

[29] A. Marshakov, A. Mironov, A. Morozov, JHEP, 11 (2009), 048, 16 pp., arXiv: 0909.3338.

[30] R. Poghossian, JHEP, 12 (2009), 038, 14 pp., arXiv: 0909.3412.

[31] Al. Zamolodchikov, Commun. Math. Phys., 96:3 (1984), 419-422.

[32] Al. Zamolodchikov, Nucl. Phys. B, 285:3 (1987), 481-503.

[33] Г. В. Белый, Изв. АН СССР. Сер. матем., 43:2 (1979), 267-276; A. Grothendieck, "Esquisse d'un programme", Geometric Galois Action, v.1: Around Grothendieck's Esquisse d'un Programme, London Mathematical Society Lecture Note Series, 242, eds. L. Schneps, P. Lochak, Cambridge Univ. Press, Cambridge, 1997, 5-48. 
[34] D. Friedan, S. Shenker, Phys. Lett. B, 175:3 (1986), 287-296; N. Ishibashi, Y. Matsuo, H. Ooguri, Modern Phys. Lett. A, 2:2 (1987), 119-132; L. Alvarez-Gaume, C. Gomez, C. Reina, Phys. Lett. B, 190:1-2 (1987), 55-62; E. Witten, Commun. Math. Phys., 113:4 (1988), 529-600; A. Morozov, Phys. Lett. B, 196:3 (1987), 325-328.

[35] A. Levin, A. Morozov, Phys. Lett. B, 243:3 (1990), 207-214.

[36] А. Д. Миронов, А. Ю. Морозов, С. М. Натанзон, 166:1 (2011), 3-27, arXiv: 0904.4227; A. Mironov, A. Morozov, S. Natanzon, J. Geom. Phys., 62:2 (2012), 148-155, arXiv: 1012.0433 .

[37] S. Kharchev, A. Marshakov, A. Mironov, A. Morozov, Internat. J. Modern Phys. A, 10:14 (1995), 2015-2051, arXiv: hep-th/9312210; А. Ю. Орлов, Д. М. Щербин, ТМФ, 128:1 (2001), 84-108; А. Ю. Орлов, ТМФ, 146:2 (2006), 222-250; A. Alexandrov, A. Mironov, A. Morozov, S. Natanzon, J. Phys. A: Math. Theor., 45:4 (2012), 045209, 10 pp., arXiv: 1103.4100 .

[38] A. Alexandrov, A. Mironov, A. Morozov, S. Natanzon, JHEP, 11 (2014), 080, 30 pp., arXiv: 1405.1395 .

[39] N. Nemkov, On fusion kernel in Liouville theory, arXiv: 1409.3537.

[40] Ал. Замолодчиков, частное сообщение.

[41] B. Ponsot, J. Teschner, Liouville bootstrap via harmonic analysis on a noncompact quantum group, arXiv: hep-th/9911110; Commun. Math. Phys., 224:3 (2001), 613-655, arXiv: math/0007097.

[42] В.Г. Книжник, А.Ю. Морозов, Писъма в ЖКЭТФ, 39:5 (1984), 202-205; Н. Levine, S. Libby, Phys. Lett. B, 150:1-3 (1985), 182-186.

[43] N. Seiberg, E. Witten, Nucl. Phys. B, 426:1 (1994), 19-52, arXiv: hep-th/9407087; Erratum, 430:2 (1994), 485-486, arXiv: hep-th/9407087; 431:3 (1994), 484-550, arXiv: hep-th/9408099.

[44] A. Gorsky, I. Krichever, A. Marshakov, A. Mironov, A. Morozov, Phys. Lett. B, 355:3-4 (1995), 466-477, arXiv: hep-th/9505035; R. Donagi, E. Witten, Nucl. Phys. B, 460:2 (1996), 299-334, arXiv: hep-th/9510101.

[45] H. Itoyama, A. Morozov, Nucl. Phys. B, 477:3 (1996), 855-877, arXiv: hep-th/9511126; Nucl. Phys. B, 491:3 (1997), 529-573, arXiv: hep-th/9512161; "Integrability and Seiberg-Witten theory", Frontiers in Quantum Field Theory (Osaka University, Osaka, Japan, 14-17 December, 1995), eds. H. Itoyama, H. Kunitomo, H. Shirokura, M. Niuomiya, World Sci., Singapore, 1996, 301-324, arXiv: hep-th/9601168; A. Gorsky, A. Mironov, "Integrable many-body systems and gauge theories", Integrable Hierarchies and Modern Physical Theories, NATO Science Series, 18, eds. H. Aratyn, A. S. Sorin, Kluwer, Dordrecht, 2001, arXiv: hep-th/0011197.

[46] Vl. S. Dotsenko, V. A. Fateev, Nucl. Phys. B, 240:3 (1984), 312-348.

[47] Б. Л. Фейгин, Д. Б. Фукс, Функи. анализ и его прил., 16:2 (1982), 47-63.

[48] M. Wakimoto, Commun. Math. Phys., 104:4 (1986), 605-609.

[49] P. Zograf, Enumeration of Grothendieck's dessins and KP hierarchy, arXiv: 1312.2538.

[50] R. Hirota, Phys. Rev. Lett., 27:18 (1971), 1192-1194; Y. Ohta, J. Satsuma, D. Takahashi, T. Tokihiro, Prog. Theoret. Phys. Suppl., 94 (1988), 210-241.

[51] G. Moore, N. Seiberg, Phys. Lett. B, 220:3 (1989), 422-430.

[52] А.В. Маршаков, А.Д. Миронов, А. Ю. Морозов, ТМФ, 164:1 (2010), 3-27, arXiv: 0907.3946.

[53] А. Д. Миронов, С.А. Миронов, А. Ю. Морозов, А.А. Морозов, ТМФ, 165:3 (2010), 503-542, arXiv: 0908.2064.

[54] K. W. J. Kadell, Compositio Math., 87:1 (1993), 5-43; Adv. Math., 130:1 (1997), 33-102; J. Kaneko, SIAM J. Math. Anal., 24:4 (1993), 1086-1110.

[55] H. Itoyama, T.Oota, Nucl. Phys. B, 838:3 (2010), 298-330, arXiv: 1003.2929. 
[56] S. Kharchev, A. Marshakov, A. Mironov, A. Morozov, S. Pakuliak, Nucl. Phys. B, 404:3 (1993), 717-750, arXiv: hep-th/9208044; A. Mironov, S. Pakuliak, Internat. J. Modern Phys. A, 8:18 (1993), 3107-3137, arXiv: hep-th/9209100; H. Awata, Y. Matsuo, S. Odake, J. Shiraishi, Soryushiron Kenkyu, 91 (1995), A69-A75, arXiv: hep-th/9503028.

[57] D. Galakhov, A. Mironov, A. Morozov, JHEP, 06 (2014), 050, 24 pp., arXiv: 1311.7069.

[58] N. Iorgov, O. Lisovyy, Yu. Tykhyy, JHEP, 12 (2013), 029, 27 pp., arXiv: 1308.4092.

[59] D. Galakhov, A. Mironov, A. Morozov, JHEP, 08 (2012), 67, 27 pp., arXiv: 1205.4998; N. Nemkov, J. Phys. A: Math. Theor., 47:10 (2014), 105401, 15 pp., arXiv: 1307.0773; M. Billo, M. Frau, L. Gallot, A. Lerda, I. Pesando, JHEP, 04 (2013), 039, 32 pp., arXiv: 1302.0686; JHEP, 11 (2013), 123, 34 pp., arXiv: 1307.6648; JHEP, 12 (2013), 029, 27 pp., arXiv: 1308.4092 .

[60] И.С. Градштейн, И.М. Рыжик, Таблищы интегралов, сумм, рядов и произведений, Физматлит, М., 1971.

[61] A. Marshakov, A. Mironov, A. Morozov, J. Geom. Phys., 61:7 (2011), 1203-1222, arXiv: 1011.4491.

[62] A. Alexandrov, A. Mironov, A. Morozov, Internat. J. Modern Phys. A, 21:12 (2006), 2481-2517, arXiv: hep-th/0412099; Fortsch. Phys., 53:5-6 (2005), 512-521, arXiv: hep-th/0412205. 